\title{
MEMORANDUM
}

No 22/ 2014

\section{Labor Supply as a Choice among Latent Jobs: Unobserved Heterogeneity and Identification}

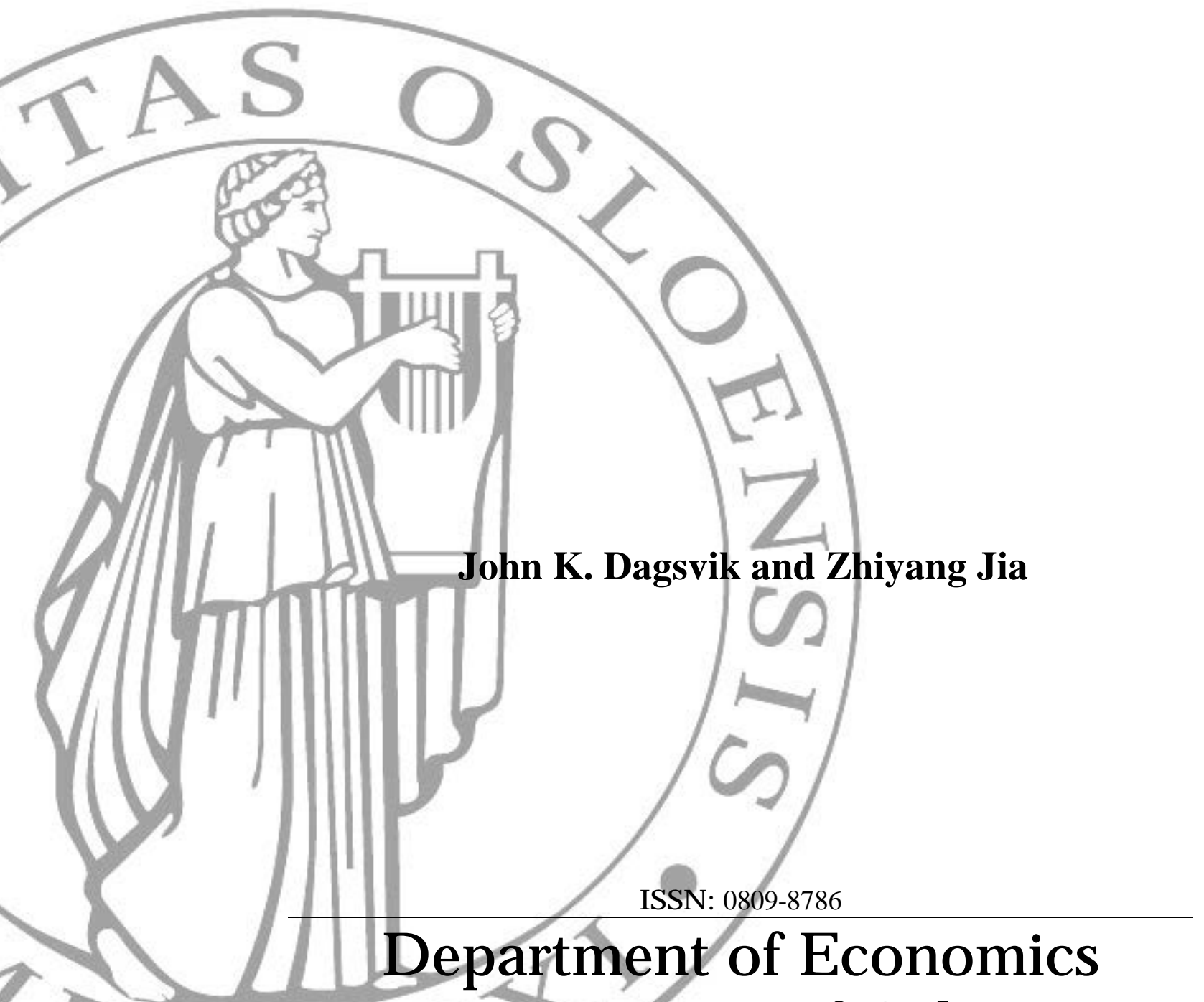

University of O slo 
This series is published by the

\section{University of Oslo \\ Department of Economics}

P. O.Box 1095 Blindern

N-0317 OSLO Norway

Telephone: + 4722855127

Fax: $\quad+4722855035$

Internet: http://www.sv.uio.no/econ

e-mail: $\quad$ econdep@econ.uio.no
In co-operation with

The Frisch Centre for Economic

Research

Gaustadalleén 21

N-0371 OSLO Norway

Telephone: $\quad$ +4722958820

Fax: $\quad$ +4722958825

Internet: $\quad$ http://www.frisch.uio.no

e-mail: $\quad$ frisch@frisch.uio.no

\section{Last 10 Memoranda}

\begin{tabular}{|c|c|}
\hline No $21 / 14$ & $\begin{array}{l}\text { Simen Gaure } \\
\text { Practical Correlation Bias Correction in Two-way Fixed Effects Linear } \\
\text { Regression }\end{array}$ \\
\hline No $20 / 14$ & $\begin{array}{l}\text { Rolf Aaberge, Tarjei Havnes and Magne Mogstad } \\
\text { A Theory for Ranking Distribution Functions }\end{array}$ \\
\hline No $19 / 14$ & $\begin{array}{l}\text { Alice Ciccone } \\
\text { Is It All About CO2 Emissions? The Environmental Effects of Tax Reform } \\
\text { for New Vehicles in Norway }\end{array}$ \\
\hline No $18 / 14$ & $\begin{array}{l}\text { Mikolaj Czajkowski, Nick Hanley and Karine Nyborg } \\
\text { Social Norms, Morals and Self-interest as Determinants of Pro- } \\
\text { environment Behaviours }\end{array}$ \\
\hline No $17 / 14$ & $\begin{array}{l}\text { Karine Nyborg } \\
\text { Reciprocal Climate Negotiators: Balancing Anger against Even More } \\
\text { Anger }\end{array}$ \\
\hline No $16 / 14$ & $\begin{array}{l}\text { Karen Evelyn Hauge and Ole Røgeberg } \\
\text { Contribution to Public Goods as Individuals versus Group Representatives: } \\
\text { Evidence of Gender Differences }\end{array}$ \\
\hline No $15 / 14$ & $\begin{array}{l}\text { Moti Michael and Daniel Spiro } \\
\text { Skewed Norms under Peer Pressure: Formation and Collapse }\end{array}$ \\
\hline No $14 / 14$ & $\begin{array}{l}\text { Daniel Spiro } \\
\text { Resource Prices and Planning Horizons }\end{array}$ \\
\hline No $13 / 14$ & $\begin{array}{l}\text { Johan Gars and Daniel Spiro } \\
\text { Uninsurance through Trade }\end{array}$ \\
\hline No $12 / 14$ & $\begin{array}{l}\text { Moti Michaeli and Daniel Spiro } \\
\text { Powerty in China as Seen from Outer Space }\end{array}$ \\
\hline
\end{tabular}

Previous issues of the memo-series are available in a PDF® format at: http://www.sv.uio.no/econ/english/research/unpublished-works/ working-papers/ 


\title{
Labor Supply as a Choice among Latent Jobs: Unobserved Heterogeneity and Identification
}

\author{
by
}

John K. Dagsvik* and Zhiyang Jia**

Memo 22/2014-v1

This version 15 September 2014

* Research Department, Statistics Norway, and the Frisch Centre of Economic Research

* *Research Department, Statistics Norway

\begin{abstract}
This paper discusses aspects of a framework for modeling labor supply where the notion of job choice is fundamental. In this framework, workers are assumed to have preferences over latent job opportunities belonging to worker-specific choice sets from which they choose their preferred job. The observed hours of work and wage is interpreted as the job-specific hours and wage of the chosen job. The main contribution of this paper is an analysis of the identification problem of this framework under various conditions, when conventional cross-section micro-data are applied.

The modeling framework is applied to analyze labor supply behavior for married/cohabiting couples using Norwegian micro data. Specifically, we estimate two model versions with in the general framework. Based on the empirical results, we discuss further qualitative properties of the model versions. Finally, we apply the preferred model version to conduct a simulation experiment of a counterfactual policy reforms.
\end{abstract}

Keywords: Labor supply, non-pecuniary job attributes, latent choice sets, random utility models, identification.

JEL classification: J22, C51.

Acknowledgments: We thank Jørgen Aasness, Thor Olav Thoresen, Terje Skjerpen and the participants in workshops at IZA, Bonn, and Statistics Norway for helpful comments. We are particularly grateful for the extensive and constructive criticism, help and suggestions by Thierry Magnac and two anonymous referees.

Email addresses: john.dagsvik@ssb.no and zhiyang.jia@ssb.no 


\section{Introduction}

In the traditional approach to labor supply modelling, individual behavior is viewed as a choice among feasible leisure and disposable income combinations. This approach has been criticized for ignoring important behavioral aspects, namely that individuals in the labor market typically have preferences over job types and may face restrictions on their choices regarding job opportunities and hours of work. Recently, the discrete choice approach to labor supply modeling has gained widespread popularity, mainly because it is much more practical than the traditional continuous approach based on marginal calculus (See Bloemen and Kapteyn, 2008). However, from a theoretical perspective, the conventional discrete choice approach represents no essential departure from the traditional approach. This is because the only new assumptions made are that the set of feasible hours of work is finite and the random components of the utility function have particular distributional properties. ${ }^{1}$

The purpose of this paper is to discuss identification and other aspects of an extended version of the conventional discrete choice model (latent job choice model) that allow for agents' preferences being dependent on non-pecuniary job attributes, as well as allowing for possible restrictions on hours of work opportunities. In the latent job choice model, originally proposed by Dagsvik (1994), the starting point is the assumption that a worker's labor supply follows from his or her job choice. More precisely, labor supply is viewed as resulting from a choice among latent job “packages”, each of which is characterized by an offered wage rate, offered hours of work and non-pecuniary (qualitative) attributes describing the nature of the job-specific tasks to be performed. This setup can be viewed as a version of Lancaster's characteristic approach - see Lancaster $(1966,1971)$ - where agents have preferences regarding not only consumption and leisure, but also job attributes. The characteristic approach is intuitively appealing because it shifts the focus to qualitative aspects of the labor market that everyday life experiences tell us are important. Examples of such latent job attributes of major importance are job-specific tasks to be performed, location, quality of the social and physical environment, etc. More recently, Farzin (2009) has discussed the effects of including non-pecuniary variables explicitly in the traditional labor supply model. He argues that ignoring such aspects of jobs can result in biased estimates and thus lead to

\footnotetext{
${ }^{1}$ Van Soest (1995) and others have proposed to introduce suitable dummies in the discrete labor supply model specification to improve the fit. However, this practice implies a non-structural model unless one interprets these dummies as part of the preference representation. In this case it means that the agent has stronger preferences for working particular hours (such as part time and full time hours) relatively to other hours.
} 
misleading policy proposals. Further related approaches are put forward by Sattinger (1993, 1995) and van Ophem et al. (1993).

The latent job choice model allows us to address neglected aspects in traditional labor supply analysis: namely that workers face important restrictions on their job choice in the labor market (Dagsvik et al., 2014). Versions of the job choice model have been introduced and applied before: see Aaberge, et al. (1995), Aaberge, et al. (1999), Dagsvik and Strøm (2004, 2006), Dagsvik and Jia (2006), Kornstad and Thoresen (2007), Di Tommaso et al. (2009), Dagsvik et al. (2011) and Dagsvik et al. (2014). This paper contains a number of new contributions. First, we analyze the identification problem in depth. The identification problem in this type of model differs from standard identification results of discrete choice models because the present model contains representations of both preferences and choice constraints. We also extend the identification analysis to the case where the distribution of offered wage rates depends on unobserved individual characteristics. The empirical literature on job choice models cited above differs greatly in this respect. Aaberge et al.(1995) and Aaberge et al. (1999) assume that there is no unobserved variation in wages across workers, so that any unobserved heterogeneity in wages is due to firm characteristics. In contrast, Dagsvik and Strøm (2006), Dagsvik and Jia (2006) and Dagsvik et al. (2011) assume that wage variation is due solely to person-specific characteristics. In this paper, we clarify the differences between these two approaches and compare their empirical performances using a sample of Norwegian married/cohabitating couples. Finally, based on the model estimates, we discuss properties of the respective models and the issue of policy simulations. In particular, we show how one can simulate the effect of changes in labor supply restrictions.

The paper is organized as follows. In Section 2 we discuss the basic structure of the modeling framework. In Section 3 we consider identification issues. In Section 4 we first report results from an empirical application. Then, we discuss how to simulate the effect of a particular reform in the restriction on working hours.

\section{The modeling framework}

Let $U(C, h, z)$ be the (ordinal) utility function of the household, where $C$ denotes household consumption (disposable income), $h$ is hours of work, $z=1,2, .$. , indexes market opportunities (jobs) and $z=-1,-2, \ldots$, indexes non-market opportunities. For a market opportunity $z$, associated hours of work and wage rate are assumed fixed and equal to $(H(z), W(z))$, where 
$H(z), z=1,2, \ldots$, takes value on a set $D$ and $W(z)$ is positive. When $z$ is negative $H(z)=0$. In addition to the economic budget constraint, there are restrictions on the set of available market opportunities faced by a specific worker. This is because there are job types for which the worker is not qualified and there may be variations in the set of job opportunities for which he or she is qualified. In addition, due to competition in the labor market, jobs for which a worker is qualified may not necessarily be available to him or her. However, the choice sets of market and non-market opportunities are unobserved by the researcher.

\section{Assumption 1}

The utility function has the structure

$$
U(C, h, z)=v(C, h) \varepsilon(z)
$$

for $\mathrm{z}=\ldots,-2,-1,1,2, \ldots$, where $v(\cdot)$ is a positive deterministic function defined on $\left[C_{0}, \infty\right) \times[0, M], \varepsilon(z)$ is a positive random taste-shifter, $C_{0} \geq 0$ is a known constant that represents subsistence consumption and $M$ is the maximum hours of work.

The random taste-shifters are supposed to capture the effect of unobserved heterogeneity in preferences over non-pecuniary attributes that affect preferences across agent and across alternatives. Whereas the functional form of the deterministic part of the utility, $v(C, h)$, can be very general, the separability condition in Assumption 1 is crucial. It may not hold in general because the error terms may depend systematically on disposable income and hours of work.

For given hours and wage rate, $h$ and $w$, the economic budget constraint is represented by $C=f(h w, I)$, where $I$ is non-labor income and $f(\cdot)$ is the net of tax function that transforms gross household income into after-tax household income. The function $f(\cdot)$ can in principle capture all details of the tax and benefit system.

The next assumption concerns the representation of the choice sets. Here, choice sets are allowed to be random in order to accommodate that they may vary across observable identical agents due to unobserved heterogeneity in opportunities. For technical convenience, we assume at the outset that the choice sets may contain an infinite number of job and nonmarket opportunities. 


\section{Assumption 2}

The taste-shifters $\{\varepsilon(z), z=. .,-2,-1\}$ associated with the available non-market opportunities and $\{\varepsilon(z), z=1,2, \ldots\}$ associated with the available jobs are realizations of two independent Poisson processes on $(0, \infty)$. The intensity of the non-market process is equal to $\varepsilon^{-2}$ and the intensity of the market process is equal to $\theta \varepsilon^{-2}$ where $\theta$ is a positive constant. The available (offered) combinations of job-specific hours of work and wage rates $\{(H(z), W(z)), z=1,2, \ldots$,$\} are independent of the corresponding taste-shifters and are$ distributed on $D \times(0, \infty)$ where $D$ is finite and contains at least 3 point, according to a joint p.d.f. $g_{1}(h) g_{2}(w \mid h)$.

Assumption 2 asserts that the taste-shifters associated with the set of available opportunities are independently scattered on the positive part of the real line but in a nonhomogeneous way. The probability that there is a job $z$ in the choice set with taste-shifter $\varepsilon(z) \in(\varepsilon, \varepsilon+\Delta \varepsilon)$ for some given positive $\varepsilon$ is (approximately) equal to $\theta \varepsilon^{-2} \Delta \varepsilon$. Since $\theta \varepsilon^{-2}$ is decreasing in $\varepsilon$, it means that the intensity is large for $\varepsilon$ close to zero. In other words, there are many available jobs that are unattractive jobs but relatively few attractive jobs available. For example, jobs and non-market opportunities may be located in another region or country.

The parameter $\theta$ is clearly a measure of job availability since a high value of $\theta$ means that there is a high probability that a job with a given level of $\varepsilon(z)$ is available. Dagsvik (1994) has demonstrated that $\theta$ can be interpreted as the ratio of the number of available market opportunities of interest (to the agent) to the number of nonmarket opportunities of interest. Note also that whereas in Assumption 1 preferences of hours are defined on the continuous set $[0, M]$, the set of feasible hours of work, $D$, is a discrete subset of $(0, M)$. The interpretation of $g_{1}(h)$ is as the probability that a job $z$ with $H(z)=h$ is available to the agent. The interpretation of $g_{2}(w \mid h) \Delta w$ is as the probability (when $\Delta w$ is small) that a job $z$ with $W(z) \in(w, w+\Delta w)$ is available to the agent, given that $H(z)=h$. In the following we shall sometimes call $\theta g_{1}(h) g_{2}(w \mid h)$ the opportunity measure and $g_{1}(h) g_{2}(w \mid h)$ the opportunity density. A motivation for this particular type of representation of the set of available opportunities is given by Dagsvik (1994). He demonstrates that the intensities of the Poisson processes must have the form given in Assumption 2 in order for the 
choice of job to satisfy the Independence from Irrelevant Alternatives (IIA) property. ${ }^{2}$ In general, the offered distribution of wage rates may depend on hours of work, similarly to Moffitt (1984). Aaronson and French (2009) have given a theoretical argument that supports this possibility.

Let $\varphi(h, w \mid I)$ denote the joint density of hours of work and wage rate of the chosen job, given non-labor income $I$, and similarly let $\varphi(0,0 \mid I)$ be the probability of not working. ${ }^{3}$

\section{Theorem 1}

If Assumptions 1 and 2 hold, then the joint p.d.f. of observing hours and wage rate combination $(h, w)$ is given by

$$
\varphi(h, w \mid I)=\frac{v(f(h w, I), h) \theta g_{1}(h) g_{2}(w \mid h)}{v(f(0, I), 0)+\theta \sum_{r \in D} \int_{0}^{\infty} v(f(r y, I), x) g_{1}(r) g_{2}(y \mid x) d y}
$$

for $h>0$, and the probability of not working is given by

$$
\varphi(0,0 \mid I)=\frac{v(f(0, I), 0)}{v(f(0, I), 0)+\theta \sum_{r \in D}^{\infty} \int_{0}^{\infty} v(f(r y, I), r) g_{1}(r) g_{2}(y \mid r) d y} .
$$

In the case with discrete conditional distribution of offered wage rates (or continuous distribution of offered hours of work) similar expressions as in (2.2a, b) follow. The only difference is that the integration is replaced by summation (or summation is replaced by integration). The proof of Theorem 1 follows from Dagsvik (1994) but for the reader's convenience a simplified version is given in Appendix A. This proof also covers the special case with degenerate distribution of offered wage rates.

For the sake of interpretation, let $B(h, w)$ be the (random) set of points of the (market) Poisson process with $H(z)=h$ and $W(z) \in(w, w+\Delta w)$, where $\Delta w$ is a small positive number. That is, $B(h, w)$ is the set of available jobs with offered hours $h$ and wage rates within $(w, w+\Delta w)$. Let $\tilde{U}(h, w)$ be the utility of the most preferred job in $B(h, w)$ that is, $\tilde{U}(h, w)=\max _{z \in B(h, w)} U(f(h w, I), h, z)$. Since $\Delta w$ is small one can show that Assumptions 1 and 2

\footnotetext{
${ }^{2}$ Dagsvik (1994) uses an equivalent additive utility specification, in which case the intensity that corresponds to $\theta \varepsilon^{-2}$ is equal to $\theta e^{-\varepsilon}$.

${ }^{3}$ For simplicity we apply the terminology of joint density function although the marginal distribution of realized hours of work is discrete.
} 
imply that the utility $\tilde{U}(h, w)$ has the same distribution as $v(f(h w, I), h) \theta g(h, w) \Delta w \tilde{\varepsilon}(h, w)$ where $\{\tilde{\varepsilon}(h, w)\}$ are positive random variables with Fréchet c.d.f. $\exp (-1 / x)$, for $x>0 .{ }^{4}$ Moreover, $\tilde{\varepsilon}\left(h^{\prime}, w^{\prime}\right)$ and $\tilde{\varepsilon}(h, w)$ are independent when $\left(h^{\prime}, w^{\prime}\right) \neq(h, w)$. Similarly, the utility of the most preferred available non-market opportunity, $\tilde{U}(0,0)$, has the same distribution as $=\max _{z \leq-1} U(f(0, I), 0, z)=v(f(0, I), 0) \tilde{\varepsilon}(0,0)$ where $\tilde{\varepsilon}(0,0)$ is a positive random variable that also is Fréchet distributed. We note that the utility $\tilde{U}(h, w)$ of the observable choice alternative $(h, w)$ can conveniently be represented by the representative utility component $\exp v(f(h w, I), h)$ weighted by $\theta g_{1}(h) g_{2}(w \mid h) \Delta w$ which captures the distribution of available market opportunities in $B(h, w)$. Apart from the property that one choice variable (wage rate) is continuous, the reformulation of the objective function above links our Poisson process formulation to the conventional theory of discrete choice.

It may be the case that many people view their number of available market opportunities of interest to be less than the number of nonmarket opportunities of interest. In addition, since $\theta$ enters the model as a factor multiplied by $v(C, h)$ it means that $\theta$ also can capture psychological “costs” of working. For these reasons one might expect that in empirical applications $\theta$ will be less than one.

We noted above that the model in Theorem 1 is consistent with an interpretation with stochastic sets of available job opportunities. There may, however, be additional unobserved heterogeneity that may enter the opportunity measure in other ways. Note that $g_{2}(w \mid h)$ may be interpreted as the conditional distribution of offered wages given offered hours of work equal to $h$. The model (2.2a, b) above assumes that $g_{2}(w \mid h)$ is the same for observable identical individuals (Assumption 2). This may be rather restrictive.

Studies on wage formations and wage dispersion have stressed the importance of unobserved individual heterogeneity: see, for example, Abowd et al. (1999) and Mortensen (2003). We will next consider a typical approach: namely that the distribution of offered wage rates for an individual depends both on observed covariates and on unobserved variables summarized in a random effect component $\eta$, which is assumed to be independent of the taste shifters $\{\varepsilon(z)\}$. It is also possible to allow $\theta$ to depend on $\eta$, i.e. $\theta=\theta(\eta)$. We shall only consider the special case where only $g_{2}(w \mid h ; \eta)$ depends on $\eta$ whereas $g_{1}(h)$ is independent

\footnotetext{
${ }^{4}$ The Fréchet distribution $\exp (-1 / x)$ in a multiplicative random utility formulation corresponds to the Gumbel distribution $\exp (-\exp (-x))$ in the additive random utility formulation.
} 
of $\eta$. The motivation for this is that we believe that hours restrictions to a large extent are determined by institutional regulations determined by negotiation between the unions of employers and workers and therefore not dependent on individual characteristics. The extended choice model is given as

$$
\varphi(h, w \mid I)=E_{\eta}\left(\frac{v(f(h w, I), h) \theta(\eta) g_{1}(h) g_{2}(w \mid h ; \eta)}{v(f(0, I), 0)+\sum_{r \in D}^{\infty} \int_{0}^{\infty} v(f(r y, I), r) \theta(\eta) g_{1}(r) g_{2}(y \mid r ; \eta) d y}\right)
$$

for $h>0$, and

$$
\varphi(0,0 \mid I)=E_{\eta}\left(\frac{v(f(0, I), 0)}{v(f(0, I), 0)+\sum_{r \in D} \int_{0}^{\infty} v(f(r y, I), r) \theta(\eta) g_{1}(r) g_{2}(y \mid r ; \eta) d y}\right)
$$

for $h=0$. Whereas the model in (2.2a, b) implies that the choice probabilities satisfy the IIA property this is not the case with (2.3a, b) due to the random effect $\eta$ in offered wage rate distribution. In the specification of (2.3a, b), the conditional distribution of offered wage rates $g_{2}(w \mid h ; \eta)$ can be represented by a offered wage rate equation which depends on $(H(z), \xi(z), \eta)$ where the latent variable $\xi(z)$ represents job-specific unobserved variation in offered wage rates across jobs given offered hours of work. A special version of the offered wage rate equation above would be an additive separable form such as

$$
\log W(z)=\alpha+\psi(H(z))+\eta+\xi(z)
$$

where $\alpha$ is a function of individual characteristics and $\eta, \xi(z)$ and $H(z)$ are assumed to be mutually independent and $\psi(H(z))$ represents the influence of the job-specific offered hours.

The case where (2.4) holds and $\xi(z)=0$ needs some further discussion. This corresponds to a model with degenerate distribution of offered wage rates, where the offered wage rates for a given individual vary only with respect to offered hours of work. Examples of studies within the conventional framework of this type include Moffitt (1984), Lundberg (1985), Biddle and Zarking (1989), Wolf (2002), and Aaronson and French (2004). These authors typically find a positive or inverse $U$ shape relationship between the wage rates and hours worked. This feature is consistent with findings in the literature where part time workers have been found to earn less than full time workers. However, the gap seems to be 
very small, once important job characteristics such as occupations are considered (Manning and Petrongolo, 2008).

Within our framework, when $\xi(z)=0$ it follows from (2.4) that for any job $z$, we have $\eta=\log W(z)-\psi(H(z))-\alpha$. Thus, given that the chosen hours of work and wage rate combination equals $(h, w)$, then it follows that any job $z$ (say) with hours of work $H(z)$ has wage rate given by $W(z)=w \exp (\psi(H(z))-\psi(h))$. Consequently, in the case with $\xi(z)=0$ (2.3a) reduces to

(2.5) $\varphi(h, w \mid I)=\frac{v(f(h w, I), h) \theta(\log w-\psi(h)-\alpha) g_{1}(h) g_{\eta}(\log w-\psi(h)-\alpha) / w}{v(f(0, I), 0)+\theta(\log w-\psi(h)-\alpha) \sum_{r \in D} v\left(f(r w \exp (\psi(r)-\psi(h)-\alpha, I), r) g_{1}(r)\right.}$ where $g_{\eta}(\cdot)$ is the p.d.f. of $\eta$. If in addition, we assume that $\psi(\cdot)=0$, i.e. the offered wage rate does not depends on offered hours of work, and that $\theta(\eta)=\theta$, (2.5) reduces to

$$
\varphi(h, w \mid I)=\frac{v(f(h w, I), h) \theta g_{1}(h)}{v(f(0, I), 0)+\theta \sum_{r \in D} v(f(r w, I), r) g_{1}(r)} \cdot \frac{g_{\eta}(\log w-\alpha)}{w}
$$

whereas in the analogous expression for the probability of not working the random effect must be integrated out. A two sector model similar to (2.6) has been applied by Dagsvik and Strøm (2006) to analyze labor supply and sectoral choice.

When $\eta=\psi(h)=0$ we get a model where offered wage rates vary across different job offers (to a given agent). This model version was applied by Aaberge et al. (1995) and Aaberge et al. (1999).

\section{Identification}

We now turn to a discussion on identification of the model. We start with the simpler case where the random effect in the wage rate equation is ruled out, i.e., the model is as presented in Theorem 1. From Theorem 1 it follows that for positive $h$,

$$
\varphi(h, w \mid I) / \varphi(0,0 \mid I)=v(f(h w, I), h) \theta g_{1}(h) g_{2}(w \mid h) / v(f(0, I), 0) .
$$

Since both $\varphi(h, w \mid I)$ and $\varphi(0,0 \mid I)$ are observable, the right hand side of (3.1) can be identified non-parametrically. However, it remains unclear if one can separately identify $v(C, h)$ and $\theta g_{1}(h) g_{2}(w \mid h)$. Essentially, the identification problem arises from the fact that observed labor supply behavior is a result of both preferences (utility function) and latent job choice constraints (opportunity measure) in our model. 
If available, information on desired hours of work could be used to identify preferences, since job choice constraints are irrelevant in this case ${ }^{5}$. Subsequently, based on the estimated preferences, one could, with this information, estimate the opportunity measures using data on actual observed behavior. In this way, both the utility function and the opportunity measure can be identified. This approach is however not straight forward because the representation of job types in stated preference surveys may not fully correspond to the variety of job offers agents face in real labor markets. On the other hand, even if panel data or independent cross-section data are available, it is hard to see how this would help to solve the identification problem in general. If, for example, preference parameters were assumed to be fixed over time, this would enable us to identify changes in the opportunity measure non-parametrically, but not the level.

In what follows we limit our discussion to the situation where only cross-section data are available: that is, we have observations on hours of work, wage, non-labor income and individual characteristics. Assumption 3 summarizes some useful regularity conditions for the deterministic part of the utility function $v(C, h)$ and the net of tax function.

\section{Assumption 3}

The deterministic part of the utility function $v(C, h)$ is continuously differentiable in $C$ and the net of tax function $f(u, I)$ is continuous, piecewise differentiable and strictly increasing in $u$ and $I$.

One important property of our setup is that non-labor income enters the utility specification in a particular manner, namely such that it can generate variation in consumption while keeping hours of work and the wage rate constant, and it enters the model only through consumption, not affecting the opportunity measure. The next theorem shows to what extent the model can be non-parametrically identified in this case.

\footnotetext{
${ }^{5}$ See for example, Bloemen (2008) and references therein for some recent studies using desired hours of work to identify preferences.
} 


\section{Theorem 2}

Assume that Assumptions 1 to 3 hold. Then $v(C, h)$ can be expressed as $v(C, h)=\zeta(C)^{r} \lambda^{*}(C, h) \delta(h)$ for $h>0$, where $\zeta(C)$ and $\lambda^{*}(C, h)$ are identified but $r$ is an unknown constant and $\delta(h)$ an unknown function of $h$.

The proof of Theorem 2 is given in Appendix A. Theorem 2 shows that even under the exclusion restriction that non-labor income does not affect the opportunity measure the model is non-parametrically unidentified. Dagsvik and Strøm (1997) show identification in an analogous model by assuming that preferences are separable in consumption and hours of work, and more importantly that fixed cost of working is observed. However, such information is rarely available.

\section{Assumption 4}

The offered wage rates and the offered hours of work are independently distributed.

\section{Theorem 3}

Assume that Assumptions 1 to 4 hold. Then the distribution of offered hours is identified and $v(C, h)=\lambda(C, h) \delta(h)$, where $\lambda(C, h)$ is identified but $\delta(h)$ is not identified.

The proof is given in Appendix A. When offered wage rates and hours of work are correlated, additional restrictions are needed to achieve identification. Note that for the purpose of simulating solely the effect of counterfactual changes in taxes and wage rates it is not necessary to identify $\delta(h)$ and $g_{1}(h)$ separately as long as $g_{1}(h)$ is kept fixed. The reason is that the effect of changes in taxed and wages only enters the model through $C$ and the opportunity density of offered wage rates.

One way to obtain full identification is to make parametric functional form assumptions about both $v(C, h)$ and $\theta g_{1}(h) g_{2}(w \mid h)$. Below, we consider one particular parametric utility specification, namely the generalized Box-Cox function given by

$$
\log v(C, h)=\gamma_{1}\left(C^{\alpha}-1\right) / \alpha+\gamma_{2}\left((1-h / M)^{\beta}-1\right) / \beta+\gamma_{3}\left(C^{\alpha}-1\right)\left((1-h / M)^{\beta}-1\right) / \alpha \beta .
$$

See Dagsvik and Strøm (2006), Dagsvik and Røine Hoff (2011) for a theoretical justification based on invariance principles. ${ }^{6}$

\footnotetext{
${ }^{6}$ See also the Errata in Dagsvik (2013).
} 


\section{Assumption 5}

There exists an interval $\left(u_{-}, u_{+}\right)$such that when $u \in\left(u_{-}, u_{+}\right)$, the marginal net of tax rate w.r.t. non-labor income $\partial f(u, I) / \partial I$ does not vary with $u$.

Assumption 5 is not particularly restrictive. In fact, most tax systems satisfy this condition.

\section{Assumption 6}

The function $\log v(C, h)$ is a generalized Box-Cox function as given in (3.2). Furthermore, there exist two points $h_{1} \neq h_{2} \in D$ such that $g_{1}\left(h_{1}\right)=g_{1}\left(h_{2}\right)$.

The following theorem summarizes the key identification results when additional functional form assumptions are made for $v(C, h)$ and $g_{1}(h) g_{2}(w \mid h)$.

\section{Theorem 4}

Assume that Assumptions 1 to 3, 5 and 6 hold. Then the model in $(2.2 a, b)$ is identified.

The proof of Theorem 4 is given in Appendix A. Theorem 4 gives sufficient conditions for the model in (2.2a, b) to be fully identified. Assumption 5 may not be necessary but is made here for convenience. As shown in the proof, under these particular functional form assumptions one can still obtain similar results as in Theorem 3 , that is, $v(C, h)$ can be identified up to an unknown function of $h$, without functional form assumptions about $g_{1}(h) g_{2}(w \mid h)$.

We shall next consider the more general setting where we allow for unobserved heterogeneity in offered wage rate, i.e. the model given by $(2.3 a, b)$. As shown below, it is necessary to introduce an exogenous variable $X$ which influences only the opportunity density.

\section{Assumption 7}

The offered wage rates are generated by

$$
\log W(z)=X b+a+\eta+\xi(z)
$$


where $X$ is a continuous covariate that does not affect preferences and can take any real value, $\xi(z)$ and $\eta$ are zero mean random variables which are independent of each other and independent of the taste-shifters, $a$ and $b$ are unknown parameters. Furthermore, $\theta$ is either a constant or has the structure

$$
\theta=\theta(a+X b+\eta)
$$

Assumption 7 asserts that the offered wage rate equation is additively separable in $a+X b, \eta$ and $\xi(z)$. The formulation in (3.3) is a special case of (2.4) with $\psi(\cdot)=0$, $\alpha=a+X b$. The parameter $a$ may depend on other individual characteristics than $X$. The motivation for the particular formulation of $\theta(\cdot)$ in Assumption 7 is that $a+X b+\eta$ is supposed to represent the effect of observed and unobserved individual ability which may affect the opportunity measure. Here, $\xi(z)$ and $\eta$ may be discrete random variables and $\xi(z)$ may also be degenerate. Although here it is sufficient for our identification results that $X$ is a scalar it could be extended to a vector. In the following we need to extend our notation of $\varphi(h, w \mid I)$ to $\varphi(h, w \mid I, X)$ to indicate that the latter p.d.f. is conditional on $X$.

\section{Assumption 8}

The function $\varphi(h, w \mid I, X) / v(f(h w, I), h))$ satisfies

$$
\int_{-\infty}^{\infty} \int_{0}^{\infty}\left(|X|^{m}|\log w|^{n} \varphi(h, w \mid I, X) / v(f(h w, I), h)\right) d w d X<\infty
$$

for $m, n=0,1,2, h \in D$ and $I>0 .^{7}$

\section{Theorem 5}

(i) If Assumptions 1 to 4, 7 and 8 hold then $v(C, h)$ is identified apart from a multiplicative term that may depend on $h, \theta(\cdot)$ is identified up to a constant and the distribution of offered wage rates conditional on the random effect is identified.

(ii) If Assumptions 1 to 4 , and 6 to 8 hold then the model in $(2.3 a, b)$ or $(2.5)$ is identified.

The proof of Theorem 5 is given in Appendix A. Theorem 5 extends the identification results of Theorems 3 and 4 to the case with a random effect in the offered wage rate

\footnotetext{
${ }^{7}$ The assumption is analogous in the case where $X$ is discrete, in which case the integral with respect to $X$ is replaced by a sum.
} 
distribution. It is worth noting that because of Assumption 4 the independence condition is still maintained in Theorem 5 in contrast to Theorem 4 where offered wage rate and hours of work are allowed to be correlated.

Theorems 2 to 5 hold also in the case where the distribution of offered wage rates is discrete or the distribution of offered hours of work is continuous. Furthermore, Theorem 5 holds when $X$ is a discrete variable or when the distribution of offered wage rates across jobs is degenerate, that is, where each agent only faces one individual specific wage rate.

\section{An empirical application}

\subsection{Model specification and estimation results.}

In this section we report results from an empirical application based on micro data from the Norwegian Labor Survey 1997. Details about variable definitions and the data, and the specification of a joint labor supply model for married couples can be found in Appendices B and C, respectively.

The systematic part of the utility function is assumed to be a generalized Box-Cox functional form, similarly to (3.2). Alternatively, we could have used a flexible polynomial specification similarly to van Soest et al. (2002). Dagsvik and Strøm $(2004,2006)$ found that the Box-Cox functional form is more or less as flexible as the polynomial specifications, and in contrast to the latter ones it is globally concave. For each spouse, we specify eight feasible annual hours of work alternatives, namely 0, 208, 624, 1,040, 1,456, 1,950, 2,340 and 2,600. The logarithm of the job availability measures $\theta_{F}$ and $\theta_{M}$ are specified as linear functions of length of schooling. The opportunity probability mass functions of offered hours, $g_{1 k}(h), k=$ $F, M$, are uniform except for peaks at full-time and part-time hours. The full-time peak corresponds to 1,950 hours annually (37.5 hours a week), while the part-time peak corresponds to 1,040 hours annually (20 hours a week). The part-time and full-time peaks in the hours distribution is supposed to capture the effect of institutional regulations on hours of work. Recall that this specification of the opportunity distribution is formally equivalent to introducing suitable dummy variables at full-time and part-time hours of work in the utility specification of the conventional discrete choice specification: see, for example, van Soest (1995). An advantage with our framework is that it provides a theoretical rationale for introducing such dummies in contrast to the conventional discrete choice labor supply model. 
A central issue in our empirical illustration is how to specify the opportunity density of offered wage rates. A fairly general class of specifications accounting for unobserved heterogeneity across both jobs and agents, as well as allowing for correlation between offered hours of work and offered wage rates, is given in (2.4). It remains, however, to prove that the model is identified in this case. Even in the case where offered hours and wage rates are independent it is a demanding task to obtain structural estimates due to measurement error in reported hours worked. In our data set, only weekly hours of work are reported but not the usual number of weeks worked during a year. Furthermore, overtime is not reported. This type of measurement error is common in data sets used for labor supply analysis. See for example Borjas (1980) and Blundell et al. (2007) for extensive discussions on how to deal with this problem in conventional labor supply models. This measurement error creates a spurious negative correlation between the observed wage rate and hours in our data (-0.22 for married women, -0.17 for married men). ${ }^{8}$ This so-called “division bias” problem poses considerable challenges for empirical analyses based on this type of data.

When offered wage rates and offered hours of work are independent, the division bias problem can be reduced by adopting the three-stage estimation procedure proposed by Dagsvik and Strøm $(2004,2006)$. This amounts to estimating a reduced-form participation probability in the first stage, and subsequently estimate wage rate equations using the results from the first stage to control for selectivity bias. In the third stage the labor supply model is estimated by the maximum likelihood method after inserting the wage rates predicted by the estimated wage equations into the model and integrating out the respective error terms. Under standard assumptions about the measurement error this leads to unbiased wage rate equations in the second stage apart from the estimate of the variance of the error terms which may be upward biased. This procedure does of course not eliminate the measurement error in hours of work but it removes the spurious negative correlation between observed hours and wage rates. Measurement error may still be a problem in the last stage maximum likelihood estimation, since it may cause misclassification of the dependent variable (individual's working hours). Hausman et al. (1998) suggest a method to control for the misspecification problem in binary choice model. However, their method is not readily applicable in our analysis. Monte Carlo evidence reported in Flood and Islam (2005) indicates that the measurement error is only a serious problem in discrete choice labor supply model when it is large.

\footnotetext{
${ }^{8}$ This error is sometimes denoted “division bias” and is a rather common problem in many typically available data sets, see for example discussion in Borjas (1980).
} 
Given Assumption 4, we have estimated two special cases of the model in (2.3a, b): Model 1 and Model 2. Using the notation of (2.5), Model 1 is based on the assumption that all observable identical individuals are assumed to face the same offered wage rate distribution across jobs $(\eta=\psi(\cdot)=0)$ whereas in Model 2 each individual faces only one offered wage, though this wage may be different for observationally identical individuals $(\xi(z)=\psi(\cdot)=0)$. Thus, the interpretation of the first stage wage rate equations is rather different in the two model versions, although the estimated equations are the same. In model 1 , the error terms in the wage rate equations measure the variation of wage rates offers across different jobs. In contrast, in Model 2, the error terms represent unobserved individual heterogeneity. ${ }^{9}$

The specification of the wage rate equations is conventional. In Model $1 \xi(z)$ is assumed to be normally distributed with zero mean and in Model $2 \eta$ is assumed to be normally distributed with zero mean. Thus, the estimated residual variances in the wage rate equations are interpreted as the variance of $\xi(z)$ and $\eta$ in Models 1 and 2, respectively. The term $\alpha$ is specified as a linear function of length of schooling, potential experience, potential experience squared and a dummy variable for marriage status. Potential experience is defined as age minus years of schooling minus 7 . The estimates of the wage rate equations are presented in Table C.1 in Appendix C. As shown in Table C.1, the selection bias in the wage rate equations is negligible.

The estimates of the two models are reported in Table C.2 in Appendix C. In both models the results imply that $\log v(C, h)$ is strictly increasing and concave in consumption and leisure. The marginal utilities of female and male leisure are increasing functions of age. The number of children has a significant effect on the marginal utility of leisure for married women. In contrast, the marginal utility of leisure for married men does not depend significantly on the number of children. This indicates that the female takes more responsibility for children within the family than the male, which is not a surprising result. The measure of the job availability for the married women, $\theta_{F}$, depends positively on the length of schooling $(S)$. Higher educational level increases the job opportunities for married women. The corresponding estimate for married men turns out not to be significant. This may be due to the fact that in our data set there are only very few married men who are out of the

\footnotetext{
${ }^{9}$ One should, in principle, interpret the estimated error terms in the Mincer type wage equations as the sum of inter- and intra-individual effects, $\xi(z)+\eta$. Although it seems technically possible to separate the contribution of these two terms, we believe that this identification hinges too much on the mathematical structure of the model and is therefore not theoretically sound, given the available information.
} 
labor force. For both genders, the value of $\theta$ is estimated to be less than 1 . Similar results are reported by Dagsvik and Strøm (2006), Di Tommaso et al. (2009) and Dagsvik et al. (2011). As discussed earlier, this can be interpreted as indicating that the number of interesting and available jobs is smaller than the number of interesting non-market opportunities. Note also that the full-time and part-time peaks in the opportunity probability mass function of hours for married men are substantially higher than the corresponding peaks for married women. The reason for this is that women seem to have stronger preferences than men for working in particular labor market sectors (such as health care and teaching) and therefore may face different choice restrictions. This could partly be due to differences in gender-specific human capital investments, which are important in shaping the job choice constraints. ${ }^{10}$

Both Model 1 and Model 2 fit the data quite well. Figures 1 and 2 display the observed and (aggregate) predicted values of participation and hours of work for each spouse based on our model. Model 2 seems to perform slightly better than Model 1. Since our model represents the behavior of couples, it seems more interesting to consider the joint distribution of hours of work rather than just the marginals. Due to the problem of thin cells, we are forced to consider joint distributions with highly aggregate hours of work intervals and combine selected hours of work intervals taking into account the fact that some hours of work alternatives are chosen by only a few households in our sample. For men, we distinguish only between the cases where they work less than full-time (0-1,456 hours a year) or work fulltime and more ( $\geq 1,950$ hours a year). For women, we distinguish between three options (not working, working less than full-time and working full-time and more). Altogether, our selected aggregation procedure implies just six cells. Figure 3 shows the predicted and observed distributions of hours of work on these six cells. As with Figures 1 and 2, the fit of both models is quite good, with Model 2 performing slightly better than Model 1.

These visual inspections of the marginal and joint distributions of hours of work can be seen as an informal and crude way to check goodness of fit of the models. Several alternative model evaluation methods are used for discrete choice models in the literature: see, for example, Train (2009). One popular summary measure analogous to the $R^{2}$ measure applied in regression analysis is McFadden's $\rho^{2}$ measure (Pseudo $R^{2}$ ), see McFadden (1973). The respective values of the loglikelihood functions are approximately equal to -5309

\footnotetext{
${ }^{10}$ In a sector-specific model, as in Dagsvik and Strøm (2006), one could obtain explicit sector-specific opportunity measures.
} 
and -5243. The corresponding $\rho^{2}$ values for the two models are 0.49 and 0.50 respectively. These results seem to be consistent with the informal check based on Figures 1 to 3 .

Figure 1. Predicted and observed marginal hours of work distributions for married women, 1997

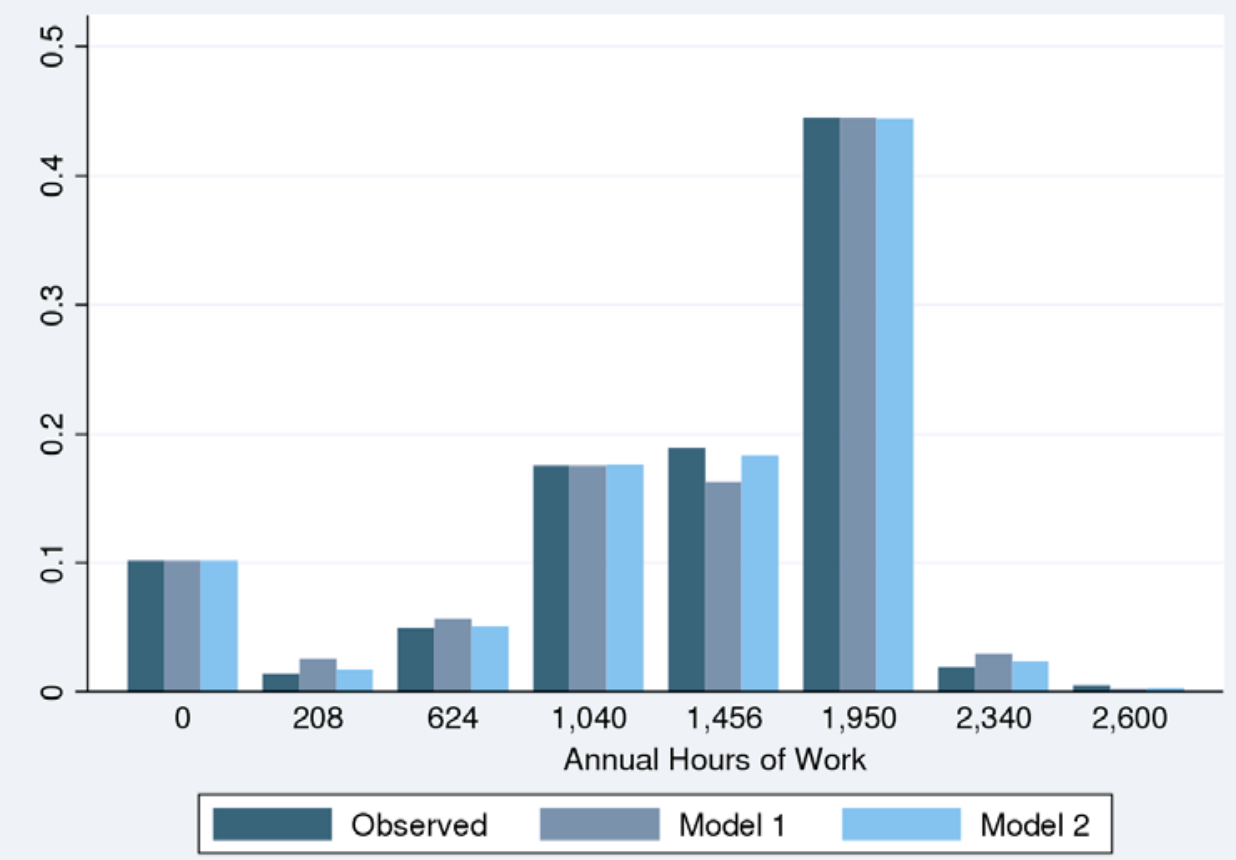

Figure 2. Predicted and observed marginal hours of work distributions for married men, 1997

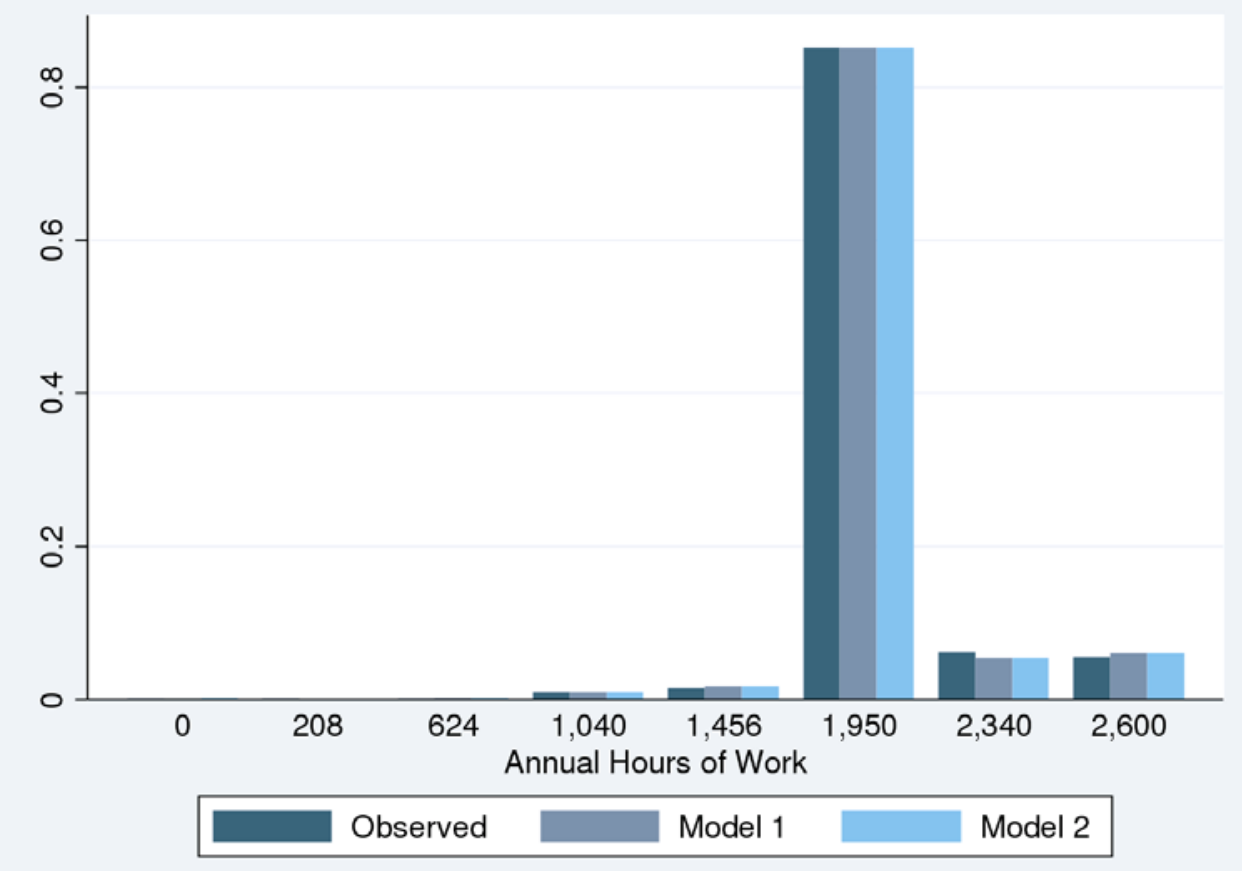


Figure 3. Predicted and observed joint hours of work distributions for couples, 1997

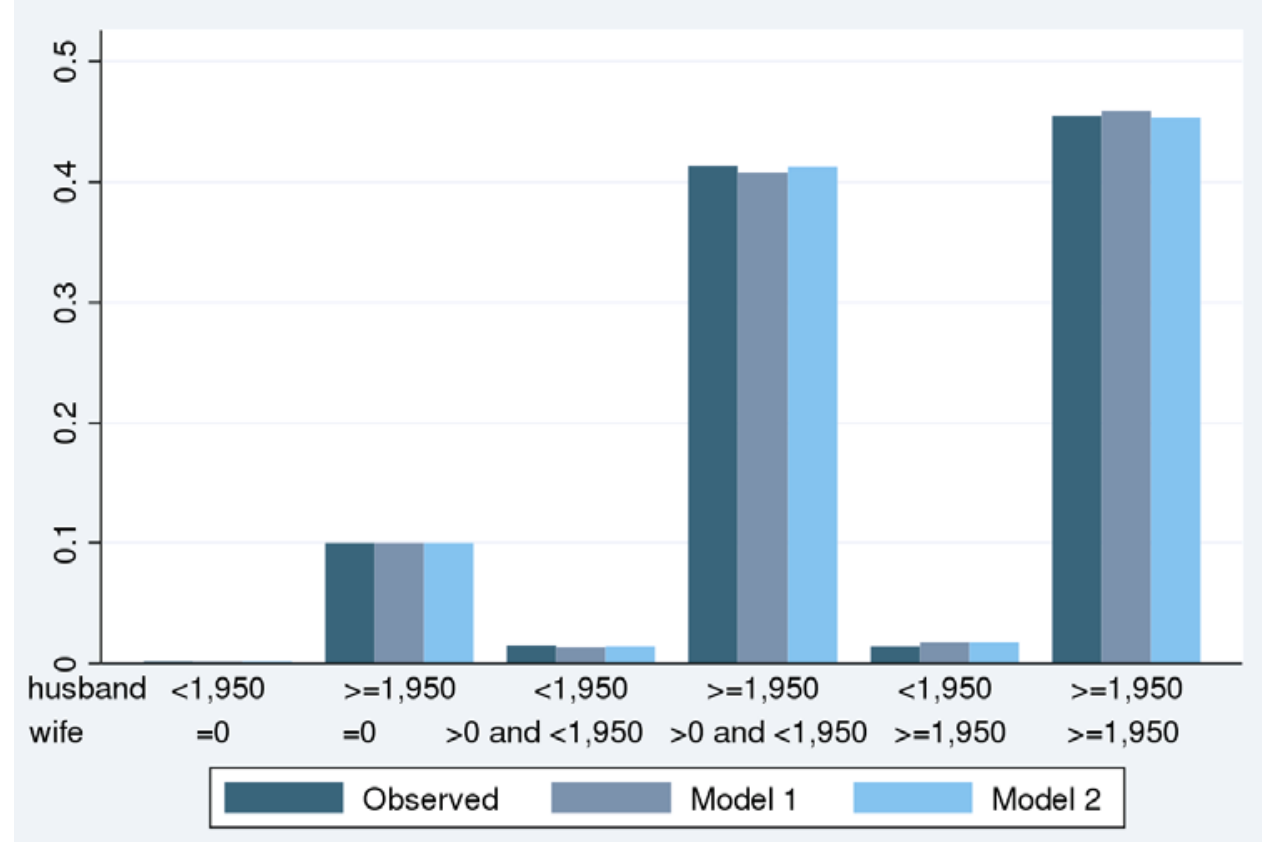

Another way of measuring goodness of fit uses tests based on comparing predicted probabilities with corresponding observed frequencies on some partitions of the outcome and exogenous variables in the tradition of the Pearson chi-square statistics. We have applied the chi-square test statistics of Andrews (1988a, b). To this end we used the same partition of cells as above (six cells). The test statistics is given as $F=N v_{N}(\widehat{\beta})^{\prime} \hat{V}^{-1} v_{N}(\widehat{\beta})$, where $v_{N}(\widehat{\beta})$ is the vector of differences between the predicted and observed frequencies on the chosen cells, and $\hat{V}$ is the estimated covariance matrix of $v_{N}(\hat{\beta}) \sqrt{N}$. In our case, the test statistics $F$ is asymptotically chi-squared distributed with 5 degrees of freedom. The test statistics for Model 1 is equal to 57.6 and for Model 2 it is equal to 10.4. Note that the 5 percent significant level for the Chi square distribution with 5 degrees of freedom is 11.07 . Thus Model 2 passes Andrew's test at 5 percent significant level, while Model 1 is far from passing the test. Thus, Model 2 fits the data better than Model 1 and we therefore select Model 2 as our maintained model. $^{11}$

\footnotetext{
${ }^{11}$ However, we do not claim that it is more important to account for inter-individual wage variations than intra-individual wage variations in this model.
} 


\subsection{Aggregate wage elasticities and "labor supply curves"}

In this section, we present selected gross wage elasticities and figures for what we call labor supply curves. We have calculated elasticities that take into account both the systematic terms and the unobservables in the model. This means that we account for how the mean of the distribution of labor supply is affected by changes in (say) gross wage levels.

There are two types of elasticities reported in the literature: see, for example, Dagsvik and Strøm (2006) and van Soest and Das (2001). One type is the so-called average elasticity, which is simply the average of the individual elasticities calculated from the formulas for expected hours of work or probability of working given the individual observed characteristics. The second is called aggregated elasticity, which is the elasticity of the aggregate (or average) response (e.g. the elasticity of the population mean hours of work).

Table 1 displays aggregated gross wage elasticities for both models. The standard errors are generated using bootstrap methods with 100 replications. We have also computed the average elasticities for both models: the estimates are quite close to the aggregated elasticities reported in Table 1. The unconditional wage elasticity of hours of work is defined as the elasticity of expected number of hours of work with respect to wage. The corresponding elasticity of hours of work conditional on working can be found by subtracting the wage elasticity of the probability of working from the unconditional wage elasticity of hours of work.

In general, both models show that the gross wage elasticities are moderate for married females and very small for married males. Model 2 seems to predict stronger labor supply response than Model 1, though the differences are not big. Using estimates from Model 2, for married females the own-wage elasticity of the probability of working is equal to 0.33 , which means that if the gross wages of married females were to increase by 5 percent (say), then the aggregate proportion of married females working would increase by around 1.5 percent. The cross wage elasticity for married women is negative and smaller in size than own wage elasticity, as found in many studies. The elasticity of the probability of working for married women with respect to both spouse's gross wage rate is equal to 0.2. This means that the proportion of married women working would increase by 1 percent, if both spouses' wage rates were increased by 5 percent.

Since the model is highly non-linear, the aggregate elasticities may cover substantial variation in elasticities across different wage levels and individual characteristics. Remember that when we have estimated the model we can compute wage elasticities conditional on 
given hypothetical wage levels, without using wage equations. To illustrate the non-linearity in the labor supply response resulting from wage changes we have plotted what we call

Table 1. Aggregated gross wage elasticities

\begin{tabular}{ccccccc}
\hline & \multicolumn{3}{c}{ Model 1 } & \multicolumn{3}{c}{ Model 2 } \\
\hline & Women's & Men’s & Both & Women’s & Men’s & Both \\
& wage & wage & wages & wage & wage & wages \\
Probability of working, & -0.006 & 0.007 & 0.003 & -0.007 & 0.010 & 0.006 \\
men & $(0.003)$ & $(0.003)$ & $(0.001)$ & $(0.003)$ & $(0.004)$ & $(0.003)$ \\
Probability of working, & 0.221 & -0.145 & 0.091 & 0.333 & -0.165 & 0.205 \\
women & $(0.015)$ & $(0.020)$ & $(0.023)$ & $(0.022)$ & $(0.022)$ & $(0.024)$ \\
Hours of work, men & -0.022 & 0.047 & 0.028 & -0.022 & 0.080 & 0.062 \\
$\quad$ (unconditional) & $(0.004)$ & $(0.007)$ & $(0.008)$ & $(0.005)$ & $(0.009)$ & $(0.009)$ \\
Hours of work, women & 0.405 & -0.268 & 0.151 & 0.618 & -0.272 & 0.381 \\
(unconditional) & $(0.025)$ & $(0.034)$ & $(0.050)$ & $(0.039)$ & $(0.038)$ & $(0.054)$ \\
\hline
\end{tabular}

Bootstrapped standard errors in parentheses.

Figure 4. Married women's expected hours of work in a typical household, by husband's wage

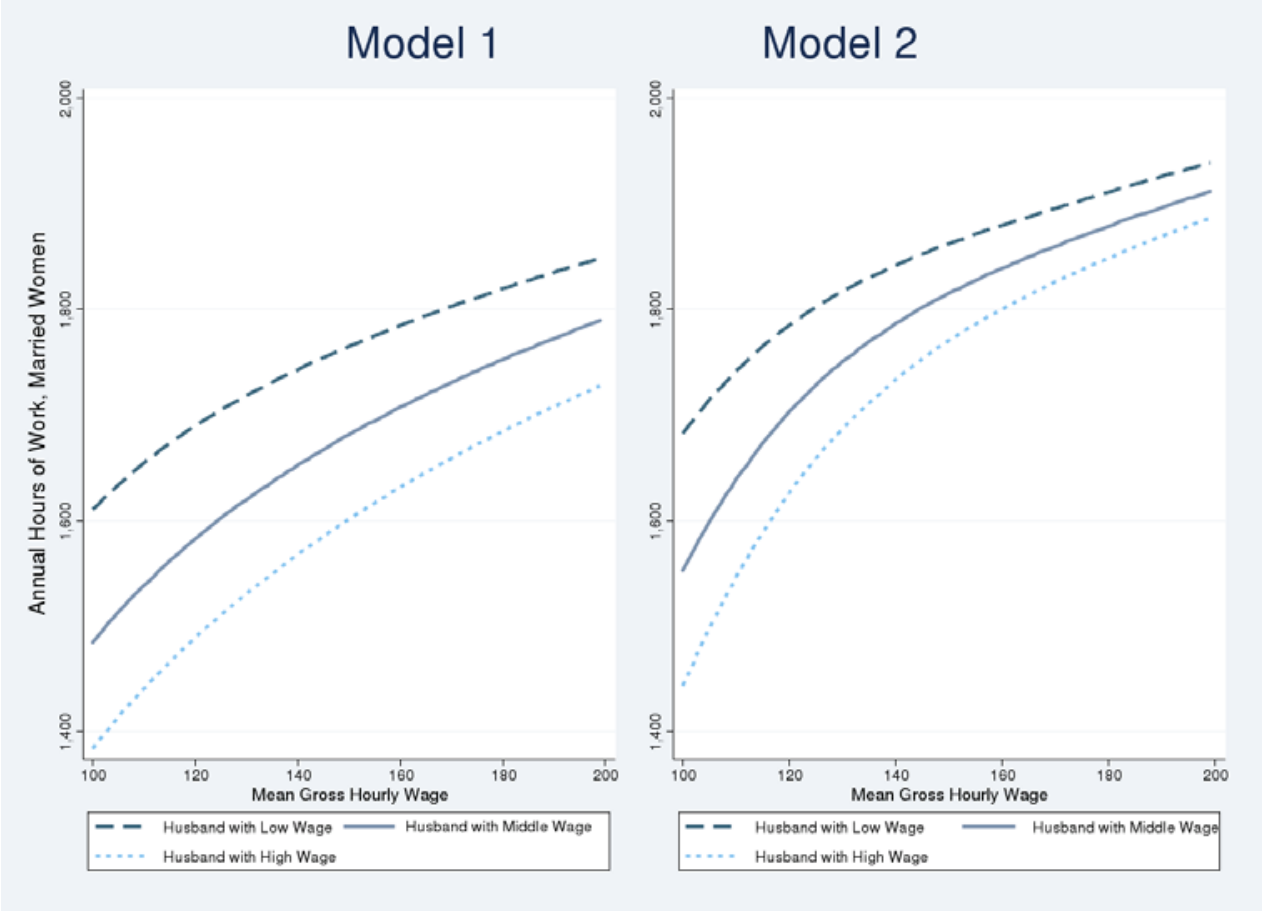

expected labor supply curves. These curves show expected hours of work as a function of the wage rates, conditional on other characteristics (non-labor income, age, family size and opportunity measure). We have generated labor supply curves for married women for a typical household, which is constructed using the sample average value of household 
characteristics given by: the household consists of a husband aged 45, a wife aged 42 and one school-age child. Both parents have 12 years of education. We look at three different scenarios: the husband's wage is high (200 NOK per hour ${ }^{12}$ ), middle (150 NOK per hour) and low (100 NOK per hour). Figures 4 shows how the expected annual hours of work of married women in both models vary with her expected gross wage level. Both models predict that labor supply elasticities decreases as married women's wage increases. However, at low wage rate levels the labor supply curves are steeper for Model 2 than for Model 1, whereas the difference is small between the two models at high wage rate levels.

\subsection{Simulation of changes in the opportunity distribution of offered hours of work}

Using our framework, we can also simulate the effect on labor supply from changing the opportunity distribution. Since our model is not an equilibrium one we can only simulate pure supply effects conditional on given the job availability measures, $\left(\theta_{F}, \theta_{M}\right)$, and the distributions of offered hours and wage rates.

In Norway, there is a high proportion of married women in part-time positions. In our sample, more than 35 percent of married women are working between 20 and 30 hours a week, while only around 40 percent are working full-time (37.5 hours a week). Many of those in part-time jobs are employed in the public sector, especially in health care. Whereas Norwegian working environment legislation opens for the possibility of voluntary part-time jobs, an important reason for the high concentration of part-time workers relates to particular institutional regulations in the public health sector. Part-time positions are sometimes the only positions offered by public health care organizations, especially in more rural areas. The proposed reform can be interpreted as a change in the opportunity distribution of hours for the women, obtained by removing the part-time peak and increasing the full-time peak (since the part-time jobs are replaced by full-time ones) while keeping the total number of available jobs unchanged (i.e. $\theta_{F}$ is unchanged). We refer to Appendix D for details.

Figure 5 displays the results from such a simulation. As we can see, there is a significant decrease in the proportion of married women who choose to work part-time, accompanied by an increase in the proportion of full-time hours of work of about a similar magnitude. In addition, we found that the corresponding labor supply of the married men changes very little with the introduction of this reform.

\footnotetext{
12 Around \$30 per hour at 1997 exchange rates.
} 
Figure 5. Hours of work for married women, before and after reform

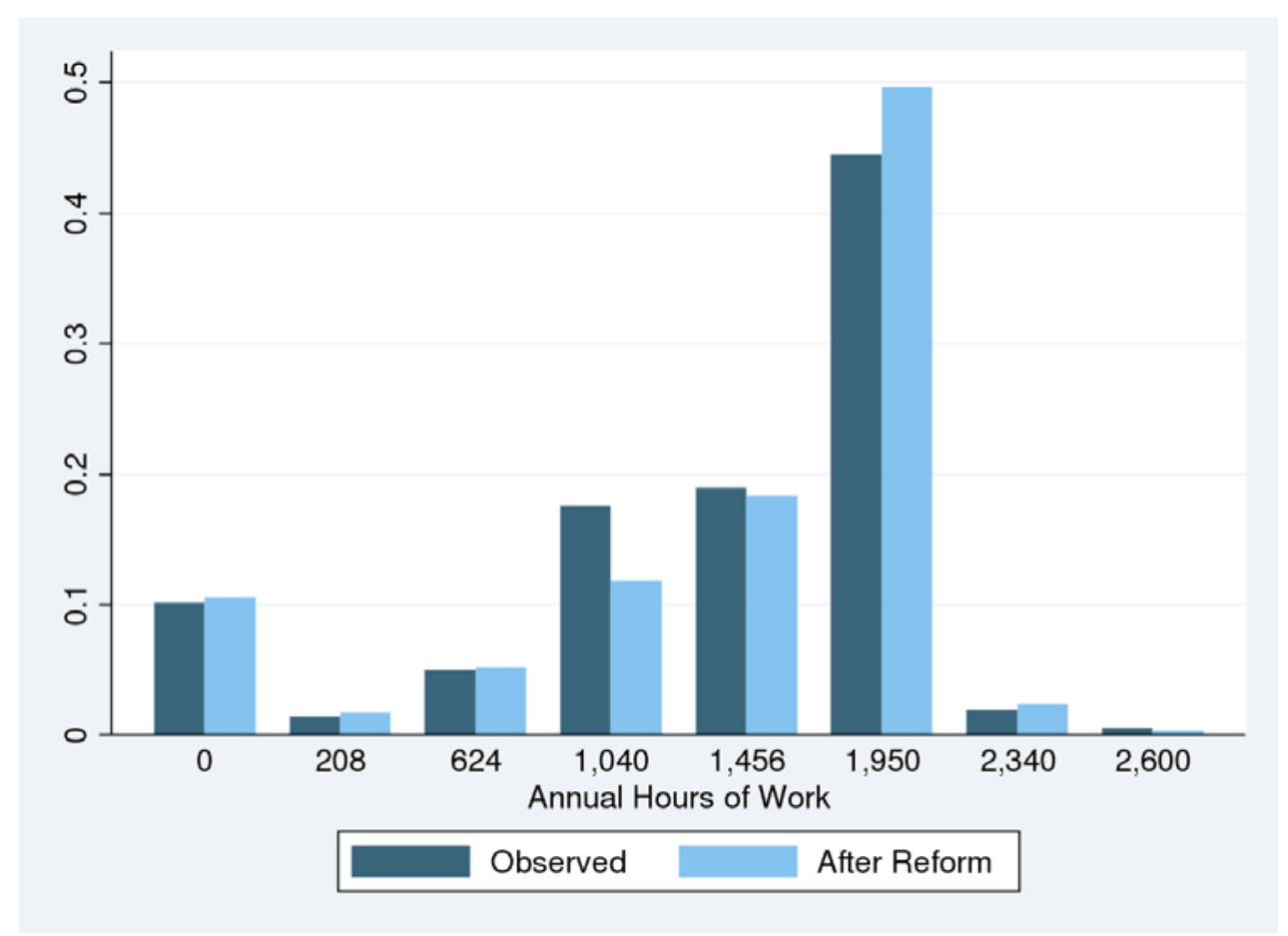

However, one needs to be careful when interpreting this result in the context of reforms specific to labor market sectors (such as the health care sector), since sector-specific preferences and restrictions are not explicitly accounted for in the model. Nevertheless, this simulation exercise clearly illustrates the advantage and potential of our modeling framework.

\section{Conclusion}

The traditional models of labor supply, being versions of the theory of consumer demand with two goods, disposable income and leisure, simplify the choice setting in labor markets. In real labor markets, agents have preferences over pecuniary as well as non-pecuniary aspects of jobs and face limited sets of job opportunities in the labor market due to competition between workers and restrictions resulting from institutional regulations on hours of work.

An essential feature of the modeling framework discussed in this paper is that it allows the researcher to accommodate restrictions on hours of work and the set of feasible jobs typically observed in many data sets. A major contribution of this paper is the analysis of identification. The standard identification results for multinomial and mixed logit models do not apply because our modeling framework contains representation of both preferences and 
latent choice constraints (opportunity measure). Although the model is in general not identified we have demonstrated how it will be identified under specific conditions even in the presence of unobserved heterogeneity in offered wage rate distribution.

We have carried out an empirical application based on micro data from Norway. In contrast to Dagsvik and Strøm (2006), where they estimated a similar model for married women given the husband's labor supply, we study the joint labor supply behavior for married couples simultaneously. We have, moreover, estimated two versions of the model based on two "extreme" assumptions about wage heterogeneity. A model with solely interindividual variation in wage rates seems to fit the data better than a model that allows only for variation in wage rates across jobs. We have computed labor supply curves to illustrate the substantial non-linearity in the labor supply responses, as function of wage rates and to illustrate how these curves depend on the two model formulations. Subsequently, we have shown how one can use the model to simulate the effect of hypothetical changes in the opportunity measure. Changes of this sort cannot be studied using conventional discrete labor supply models.

The data we have applied are not ideal due to measurement error in the hours of work observations. We are currently working on establishing another data set with much less measurement error in hours which hopefully will be helpful for studying the relationship between the offered wage rates and offered hours within a labor supply modeling framework. 


\section{References}

Aaberge, R, U. Columbino and S. Strøm (1999): Labor Supply in Italy: an Empirical Analysis of Joint Household decisions, with Taxes and Quantity Constraints. Journal of Applied Econometrics, 14, 403-422.

Aaberge, R., J. K. Dagsvik and S. Strøm (1995): Labor Supply Responses and Welfare Effects of Tax Reforms. Scandinavian Journal of Economics, 97, 635-659.

Abowd, J., F. Kramarz and D. Margolis (1999): High Wage Workers and High Wage Firms. Econometrica, 67, 251-334.

Andrews, D. W. K. (1988a): Chi-square diagnostic tests for econometric models: Introduction and applications. Journal of Econometrics, 37, 135-156.

Andrews, D. W. K. (1988b): Chi-Square Diagnostic Tests for Econometric Models: Theory. Econometrica, 56, 1419-1453.

Aaronson, D. and E. French (2004): The Effect of Part-Time Work on Wages: Evidence from the Social Security Rules. Journal of Labor Economics, 22, 329-352.

Aaronson, D. and E. French (2009): The Effect of Progressive Taxation on Labor Supply with Hours and Wages are Jointly Determined. Journal of Human Resources, 44, 386-408.

Borjas, G (1980): The Relationship between Wages and Weekly Hours of Work: The Role of the Division Bias. Journal of Human Resources, 15, 409-423.

Biddle, J. and G. Zarkin (1989): Choice among Wage-Hours Packages: an Empirical Investigation of Male Labor Supply. Journal of Labor Economics, 7, 415-437.

Bloemen, H. (2008): Job Search, Hours Restrictions, and Desired Hours of Work. Journal of Labor Economics, 26, 137-179.

Bloemen, H. and A. Kapteyn (2008): The Estimation of Utility Consistent Labor Supply Models by Means of Simulated Scores. Journal of Applied Econometrics, 23, 395-422.

Blundell, R., MaCurdy, T. and Meghir, C. (2007): Labor Supply Models: Unobserved Heterogeneity, Nonparticipation and Dynamics, in: J.J. Heckman \& E.E. Leamer (ed.), Handbook of Econometrics, Volume 6A, Elsevier.

Dagsvik, J. K. (1994): Discrete and Continuous Choice, Max-stable Processes and Independence from Irrelevant Attributes. Econometrica, 62, 1179-1205.

Dagsvik, J. K. (2013): Justification of Functional Form Assumptions in Structural Models: a Correction. Theory and Decision, 75, 79-83. 
Dagsvik, J. K. and Z. Jia (2006): Labor Supply as a Choice among Latent Job Opportunities. A Practical Empirical Approach, Discussion Papers 481, Research Department of Statistics Norway.

Dagsvik, J. K. and S. Røine Hoff (2011): Justification of Functional Form Assumptions in Structural Models: Applications and Testing of Qualitative Measurement Axioms. Theory and Decision, 70, 215-254.

Dagsvik, J. K. and S. Strøm (1997): A Framework for Labor Supply Analysis in the Presence of Complicated Budget Restrictions and Qualitative Opportunity Aspects. Memorandum from Department of Economics, University of Oslo, No 22.

Dagsvik, J. K. and S. Strøm (2004): Sectoral Labor Supply, Choice Restrictions and Functional Form. Discussion Paper no. 388, Statistics Norway, Oslo.

Dagsvik, J. K. and S. Strøm (2006): Sectoral Labor Supply, Choice Restrictions and Functional Form. Journal of Applied Econometrics, 21, 803-826.

Dagsvik J. K, Z. Jia, T. Kornstad and T. Thoresen (2014): Theoretical and Practical Arguments for Modeling Labor Supply as a Choice among Latent Jobs, Journal of Economic Surveys. 28, 134-151.

Dagsvik J. K., Z. Jia, K. Orsini and G. van Camp (2011): Subsidies on Low-skilled Workers' Social Security Contributions: the Case of Belgium. Empirical Economics, 40, 779-806.

Di Tommaso, M. L., E. M. Sæther and S. Strøm (2009): Nurses Wanted. Journal of Health Economics, 28, 748-757.

Farzin, Y. H. (2009): The Effect of Non-pecuniary Motivations on Labor Supply. The Quarterly Review of Economics and Finance, 49, 1236-1259.

Flood, L., and N. Islam (2005): A Monte Carlo Evaluation of Discrete Choice Labour Supply Models, Applied Economics Letters, 12, 263-266.

Hausman, J. A., J. Abrevaya and F. M. Scott-Morton (1998): Misclassification of the Dependent Variable in a Discrete-response Setting, Journal of Econometrics 87, 239-269.

Kornstad, T. and T. O. Thoresen (2007): A Discrete Choice Model for Labor Supply and Child Care. Journal of Population Economics, 20, 781-803.

Lancaster, K. (1966): A New Approach to Consumer Demand. Journal of Political Economy, 74, 132-157.

Lancaster, K. (1971): Consumer Demand: A New Approach. Columbia University Press: New York.

Lundberg, S. (1985): Tied Wage-Hours Offers and the Endogeneity of Wages. Review of Economics and Statistics, 83, 638-646. 
Manning, A. and B. Petrongolo (2008): The Part-Time Pay Penalty for Women in Britain. Economic Journal, 118, F28-F51.

McFadden, D. (1973): Conditional Logit Analysis of Qualitative Choice Behavior. In P. Zarmebka (ed.), Frontiers in Econometrics, Academic Press, New York.

Moffitt, R. (1984): The Estimation of a Joint Wage-Hours Labor Supply Model. Journal of Labor Economics, 2, 550-566.

Mortensen, D. T. (2003): Wage Dispersion: Why are Similar Workers Paid Differently? MIT Press: MA, USA.

Resnick, S. I. (1987): Extreme Values, Regular Variation, and Point Processes. SpringerVerlag: Berlin.

Sattinger, M. (1993): Assignment Models of the Distribution of Earnings. Journal of Economic Literature, 31, 831-880.

Sattinger, M. (1995): Search and the Efficient Assignment of Workers to Jobs. International Economic Review, 36, 283-302.

Train, K. (2009): Discrete Choice Methods with Simulation. Cambridge University Press, New York.

Van Ophem, H., J. Hartog and W. Vijverberg (1993): Job Complexities and Wages. International Economic Review, 34, 853-873.

Van Soest, A. (1995): Structural Models of Family Labor Supply. A Discrete Choice Approach. Journal of Human Resources, 30, 63-88.

Van Soest, A. and M. Das (2001): Family Labor Supply and Proposed Tax Reforms in the Netherlands. De Economist, 149, 191-218.

Van Soest, A., M. Das and X. Gong (2002): A Structural Labour Supply Model with Flexible Preferences. Journal of Econometrics, 107, 345-375.

Wolf, E. (2002): Lower Wage Rates for Fewer Hours? A Simultaneous Wage-hours Model for Germany. Labour Economics, 9, 643-663. 


\section{Appendix A}

\section{Proof of Theorem 1:}

From Assumptions 1 and 2 and Proposition 3.8 in Resnick (1987, p. 135) it follows that the job attributes $\Upsilon=\{(H(z), W(z), \varepsilon(z)), z=1,2, \ldots$,$\} can be viewed as realizations from a three-$ dimensional Poisson process on $\Psi=D \times(0, \infty) \times(0, \infty)$ with intensity measure $g_{1}(h) g_{2}(w \mid h) \theta \varepsilon^{-2} d w d \varepsilon$. Let $B=B_{1} \times B_{2}$ where $B_{1} \subset D$, and $B_{2}$ is a Borel set on $(0, \infty)$. Let

$$
U(z)=\left\{\begin{array}{ll}
v(f(H(z) W(z), I), H(z)) \varepsilon(z), & z=1,2, \ldots, \\
v(f(0, I), 0) \varepsilon(z) & z=-1,-2, \ldots
\end{array},\right.
$$

and define

$$
U_{B}=\max _{(H(z), W(z)) \in B \cap Y} U(z) .
$$

(Here it is understood that maximization is taken with respect to $z$ in $B$, for a given realization of the Poisson Process). Evidently, $U_{B}$ is the highest utility the agent can attain given that hours of work and wage rates are restricted to $B$. Note that if two sets $A$ and $B$ are disjoint sets in $\Psi$ then $U_{A}$ and $U_{B}$ are independent random variables, since the points of the Poisson process are independently scattered. Let $\Omega$ be the set defined by

$$
\Omega(u)=\{(h, w, \varepsilon) \in \Psi:(h, w) \in B, v(f(h w, I), h) \varepsilon>u\}
$$

and let $N(\Omega(u))$ be the number of points of the three-dimensional Poisson process within $\Omega(u)$. By the properties of the Poisson process it follows that

$$
P(N(\Omega(u))=n)=\frac{\Lambda(\Omega(u))^{n} \exp (-\Lambda(\Omega(u)))}{n !}
$$

where

$$
\begin{gathered}
\Lambda(\Omega(u))=E N(\Omega(u))=\sum_{x \in B_{1}} \int_{y \in B_{2}, v} \int_{(f(x y, I), x)} g_{1}(x) g_{2}(y \mid x) \theta \varepsilon^{-2} d y d \varepsilon \\
=\theta u^{-1} \sum_{x \in B_{1}} \int_{y \in B_{2}} v(f(x y, I), x) g_{1}(x) g_{2}(y \mid x) d y .
\end{gathered}
$$

Now consider the probability that $U_{B} \leq u$. Evidently, it must be the case that

(A.1) $P\left(U_{B} \leq u\right)=P$ ( There are no points of the Poisson process within $\Omega(u)$ )

$$
=P(N(\Omega(u))=0)=\exp (-\Lambda(\Omega(u)))=\exp \left(-\theta u^{-1} \sum_{x \in B_{1}} \int_{B_{2}} v(f(x y, I), x) g_{1}(x) g_{2}(y \mid x) d y\right) .
$$


Hence, we have proved that $U_{B}$ is distributed according to the Fréchet distribution.

Now we specialize $B$ to $B_{1}=\{h\}$ and $B_{2}=(0, w]$, and let $C=D \times(0, \infty) \backslash B$. Since $B$ and $C$ are disjoint, it follows, as mentioned above that $U_{B}$ and $U_{C}$ are independent and Fréchet distributed. In particular it follows from (A.1) that

$$
P\left(U_{B} \leq u\right)=\exp \left(-\theta u^{-1} \int_{0}^{w} v(f(h y, I), h) g_{1}(h) g_{2}(y \mid h) d y\right)
$$

and

$$
P\left(U_{C} \leq u\right)=\exp \left(-\theta u^{-1}\left(\sum_{D \backslash B_{1}} \int_{(0, \infty) \backslash B_{2}} v(f(x y, I), x) g_{1}(x) g_{2}(y \mid x) d y\right)\right) .
$$

Let

$$
U_{0}=\max _{z \leq-1} U(z)=v(f(0, I), 0) \max _{z \leq-1} \varepsilon(z) .
$$

Since $\{\varepsilon(z), z=. .,-2,-1\}$ are realizations of independent Poisson processes on $(0, \infty)$ with intensity $\varepsilon^{-2}$, it follows similarly to the demonstration above that $U_{0}$ also is Fréchet distributed and independent of $U_{B}$ and $U_{C}$ with

$$
P\left(U_{0} \leq u\right)=\exp \left(-u^{-1} v(f(0, I), 0)\right) .
$$

Then the probability that the agent shall choose a job with hours of work equal to $h$ and wage less than or equal to $w$, among all available work options and non-market options, can be expressed as $P\left(U_{B}>\max \left(U_{0}, U_{C}\right)\right)$. From (A.2-A.4) we thus obtain that

$$
P\left(U_{B}>\max \left(U_{0}, U_{C}\right)\right)=\frac{\theta \int_{0}^{w} v(f(h y, I), h) g_{1}(h) g_{2}(y \mid h) d y}{v(f(0, I), 0)+\theta \sum_{r \in D} \int_{0}^{\infty} v(f(r y, I), y) g_{1}(r) g_{2}(y \mid r) d y} .
$$

The corresponding p.d.f. (2.2a) is found by differentiating the expression above with respect to $w$. Due to the adding-up restriction the probability for the nonworking option follows immediately from the expression above and is equal to

$$
P\left(U_{0}>\max \left(U_{B}, U_{C}\right)\right)=\frac{v(f(0, I), 0)}{v(f(0, I), 0)+\theta \sum_{r \in D} \int_{0}^{\infty} v(f(r y, I), y) g_{1}(r) g_{2}(y \mid r) d y} .
$$

Hence, we have proved (2.2b). The proofs in the cases where the distribution of offered hours of work is continuous or the distribution of offered wage rates is discrete are similar. 
This concludes the proof.

Q.E.D.

\section{Proof Theorem 2:}

Let $F_{k}^{\prime}(x)$ denote the partial derivative with respect to the $k$-th component of $x$ of a function $F(x)$. By assumption 3, $f(u, I)$ is invertible in $u$ for a given $I$ except at a finite number of points. Thus, $\kappa(C, I)$ determined by $C=f(\kappa(C, I), I)$ is a well defined function and equals the wage income which is needed to achieve disposable income $C$ at given non-labor income level $I$.

From (2.2a, b) we have that

$$
\frac{v(f(h w, I), h) \theta g(h, w)}{v(f(0, I), 0)}=\frac{\varphi(h, w \mid I)}{\varphi(0,0 \mid I)}
$$

where $\varphi(h, w \mid I)$ is the joint density of observed hours and wage rate. By taking the logarithm transformation of (A.5) and differentiating with respect to $I$ we obtain

$$
\frac{v_{1}^{\prime}(f(h w, I), h) f_{2}^{\prime}(h w, I)}{v(f(h w, I), h)}-\frac{v_{1}^{\prime}(f(0, I), 0) f_{2}^{\prime}(0, I)}{v(f(0, I), 0)}=\frac{\varphi_{3}^{\prime}(h, w \mid I)}{\varphi(h, w \mid I)}-\frac{\varphi_{3}^{\prime}(0,0 \mid I)}{\varphi(0,0 \mid I)} .
$$

By inserting $w h=\kappa(C, I)$ into (A.6) and rearranging, (A.6) becomes

$$
\frac{v_{1}^{\prime}(C, h)}{v(C, h)}=\frac{\partial \log v(C, h)}{\partial C}=\frac{r(I)}{f_{2}^{\prime}(\kappa(C, I), I)}+\Gamma(C, h, I) .
$$

where

$$
\Gamma(C, h, I)=\left(\frac{\varphi_{3}^{\prime}(h, \kappa(C, I) / h \mid I)}{\varphi(h, \kappa(C, I) / h \mid I)}-\frac{\varphi_{3}^{\prime}(0,0 \mid I)}{\varphi(0,0 \mid I)}\right) \frac{1}{f_{2}^{\prime}(\kappa(C, I), I)} .
$$

and

$$
r(I)=v_{1}^{\prime}(f(0, I), 0) f_{2}^{\prime}(0, I) / v(f(0, I), 0) .
$$

By integrating (A.7) with respect to consumption we get that

$$
\log v(C, h)-\log v\left(C_{0}, h\right)=r(I) \int_{C_{0}}^{C} \frac{d z}{f_{2}^{\prime}(\kappa(z, I), I)}+\int_{C_{0}}^{C} \Gamma(z, h, I) d z .
$$

Let

$$
\log \zeta(C, I)=\int_{C_{0}}^{C} \frac{d z}{f_{2}^{\prime}(\kappa(z, I), I)} \text { and } \log \lambda^{*}(C, h, I)=\int_{C_{0}}^{C} \Gamma(z, h, I) d z .
$$

With this notation we obtain from (A.10) that

$$
v(C, h)=\zeta(C, I)^{r(I)} \lambda^{*}(C, h, I) v\left(C_{0}, h\right) .
$$


for $h>0$. We note that the left-hand side of (A.11) does not depend on $I$. We can therefore keep $I$ fixed and equal to any given $I^{*}$ (say) in the expression on the right hand side. Since $f(u, I)$ is known and $\Gamma(C, h, I)$ is identified due to (A.8), both $\zeta\left(C, I^{*}\right)$ and $\lambda^{*}\left(C, h, I^{*}\right)$ are identified for positive $h$. However, even when $I^{*}$ has been fixed, (A.9) cannot be used to determine $r\left(I^{*}\right)$ since $v(C, 0)$ is not identified.

When $I^{*}$ has been fixed, we can suppress $I^{*}$ in the notation and write $\zeta(C)=\zeta\left(C, I^{*}\right), \lambda^{*}(C, h)=\lambda^{*}\left(C, h, I^{*}\right), r=r\left(I^{*}\right)$ and $v\left(C_{0}, h\right)=\delta(h)$ in (A.11), which completes the proof.

Q.E.D.

\section{Proof of Theorem 3:}

By assumption 4, $g_{2}(w \mid h)=g_{2}(w)$. From (2.2a) and (A.11) it then follows that for $I>0$, $h \neq h^{*} \in D$,

$$
\frac{\varphi(h, w \mid I)}{\varphi\left(h^{*}, w \mid I\right)}=\frac{v(f(h w, I), h) g_{1}(h)}{v\left(f\left(h^{*} w, I\right), h\right) g_{1}\left(h^{*}\right)}=\frac{\zeta(f(h w, I), I)^{r(I)} \lambda^{*}(f(h w, I), h, I) \delta(h) g_{1}(h)}{\zeta\left(f\left(h^{*} w, I\right), I\right)^{r(I)} \lambda^{*}\left(f\left(h^{*} w, I\right), h^{*}, I\right) \delta\left(h^{*}\right) g_{1}\left(h^{*}\right)}
$$

which implies that for $w_{1} \neq w_{2}$

$$
\begin{aligned}
& \log \left(\frac{\varphi\left(h, w_{1} \mid I\right) \lambda^{*}\left(f\left(h^{*} w_{1}, I\right), h^{*}, I\right)}{\varphi\left(h^{*}, w_{1} \mid I\right) \lambda^{*}\left(f\left(h w_{1}, I\right), h, I\right)} \cdot \frac{\varphi\left(h^{*}, w_{2} \mid I\right) \lambda^{*}\left(f\left(h w_{2}, I\right), h, I\right)}{\varphi\left(h, w_{2} \mid I\right) \lambda^{*}\left(f\left(h^{*} w_{2}, I\right), h^{*}, I\right)}\right) \\
& =r(I) \cdot \log \left(\frac{\zeta\left(f\left(h w_{1}, I\right), I\right)}{\zeta\left(f\left(h^{*} w_{1}, I\right), I\right)} \cdot \frac{\zeta\left(f\left(h^{*} w_{2}, I\right), I\right)}{\zeta\left(f\left(h w_{2}, I\right), I\right)}\right) .
\end{aligned}
$$

From (A.12) we see that $r(I)$ is identified. Thus $\lambda(C, h)$ defined by $\lambda(C, h)=\zeta(C, I)^{r(I)} \lambda^{*}(C, h, I)$ is identified and (A.11) implies that $\lambda(C, h)$ does not depend on $I$. Hence, $v(C, h)=\lambda(C, h) \delta(h)$ for positive $h$. We thus realize that $v(C, h)$ is identified for positive $h$ up to a multiplicative term $\delta(h)$ that is solely a function of hours of work.

Consider next the case with $h=0$. From (A.9) we have

$$
\frac{\partial \log v(f(0, I), 0)}{\partial I}=r(I)
$$

The right hand side of (A.13) is known, since $r(I)$ is identified by (A.12). By integrating (A.13) with respect to $I$ we realize that $\log v(f(0, I), 0)$ is determined for each positive $I$ up to an additive constant. But this means that $v(C, 0)$ is identified up to a multiplicative constant. In the case where the distribution of offered wage rates is not degenerate we get from (2.2a) and the results above we have 


$$
g_{2}(w)=g_{2}\left(w^{*}\right) \frac{\varphi(h, w \mid I) / \lambda(f(h w, I), h)}{\varphi\left(h, w^{*} \mid I\right) / \lambda\left(f\left(h w^{*}, I\right), h\right)} .
$$

By integrating (A.14) (or summing in the case with discrete offered wage rates) with respect to positive $w$ it follows (because the left hand side is a p.d.f. function) that

$$
1=\frac{g_{2}\left(w^{*}\right)}{\varphi\left(h, w^{*} \mid I\right) / \lambda\left(f\left(h w^{*}, I\right), h\right)} \int_{0}^{\infty}(\varphi(h, y \mid I) / \lambda(f(h y, I), h)) d y
$$

which together with (A.14) yields

$$
g_{2}(w)=\frac{\varphi(h, w \mid I) / \lambda(f(h w, I), h)}{\int_{0}^{\infty}(\varphi(h, y \mid I) / \lambda(f(h y, I), h)) d y}
$$

which shows that $g_{2}(w)$ is identified. The proof is thus complete.

Q.E.D.

\section{Proof of Theorem 4:}

Since by assumption $5, f_{2}^{\prime}(u, I)$ is constant in $u$ within certain intervals, one can find an interval $\left(C_{-}, C_{+}\right)$(say), a non-labor income $I^{*}$ and a constant $d \neq 0$, such that $f_{2}^{\prime}\left(\kappa\left(C, I^{*}\right), I^{*}\right)=d$, for $C \in\left(C_{-}, C_{+}\right)$where $\kappa(C, I)$ is defined as in the proof above.

By assumption $6, \log v(C, h)$ has the generalized Box-Cox functional form given in (3.2). In the interval $\left(C_{-}, C_{+}\right)$, (A.7) can be rewritten as

$$
\frac{\partial \log v(C, h)}{\partial C}=\frac{r\left(I^{*}\right)}{d}+\Gamma\left(C, h, I^{*}\right)
$$

where $r\left(I^{*}\right)$ is unknown. It then follows from (3.2) and differentiation of (A.16) with respect to $C$ that

$$
\frac{\partial^{2} \log v(C, h)}{\partial C^{2}}=\gamma_{1}(\alpha-1) C^{\alpha-2}\left(1+\frac{\gamma_{3}}{\gamma_{1}} \frac{(1-h / M)^{\beta}-1}{\beta}\right)=\Gamma_{1}^{\prime}\left(C, h, I^{*}\right) .
$$

For the case $\beta=0$, the term $\left((1-h / M)^{\beta}-1\right) / \beta$ in (A.17) is replaced by its limit $\log (1-h / M)$. Let $C^{*} \in\left(C_{-}, C_{+}\right)$be a value of disposable income different from $C$. Then (A.17) implies that

$$
\left(\frac{C}{C^{*}}\right)^{\alpha-2}=\frac{\Gamma_{1}^{\prime}\left(C, h, I^{*}\right)}{\Gamma_{1}^{\prime}\left(C^{*}, h, I^{*}\right)}
$$

which identifies $\alpha$. Let $h_{0}=\min \{h \mid h \in D\}$ and define $x=\log \left((M-h) /\left(M-h_{0}\right)\right)$. We see immediately that $x \leq 0$. For $x \leq 0$ define 


$$
F(x)=\frac{\Gamma_{1}^{\prime}\left(C, M\left(1-e^{x}\right)+e^{x} h_{0}, I^{*}\right)-\Gamma_{1}^{\prime}\left(C, h_{0}, I^{*}\right)}{C^{\alpha-2}}
$$

From (A.17) it follows that

$$
F(x)=\left\{\begin{array}{cc}
(\alpha-1) \gamma_{3} \frac{\left(e^{\beta x}-1\right)}{\beta} & \text { if } \beta \neq 0, \\
(\alpha-1) \gamma_{3} x & \text { otherwise. }
\end{array}\right.
$$

Let

$$
G\left(\beta \mid x_{1}, x_{2}\right)=\left(e^{\beta x_{1}}-1\right) /\left(e^{\beta x_{2}}-1\right)
$$

for $\beta \neq 0$ and $G\left(0 \mid x_{1}, x_{2}\right)=x_{1} / x_{2}$, for , $x_{1}<0, x_{2}<0$. It is easy to show that $G$ is differentiable as a function of $\beta$ on the real line (also at $\beta=0$ ). From (A.19) we obtain that

$$
G\left(\beta \mid x_{1}, x_{2}\right)=\frac{F\left(x_{1}\right)}{F\left(x_{2}\right)}
$$

for suitable $x_{1}<x_{2}<0$. We shall show that there is at most one $\beta$ that satisfies (A.20). Note first that

$$
\frac{\partial G\left(\beta \mid x_{1}, x_{2}\right)}{\partial \beta}=\frac{x_{1} x_{2} e^{\beta\left(x_{1}+x_{2}\right)}}{\left(e^{\beta x_{2}}-1\right)^{2}}\left(\frac{1-e^{-\beta x_{2}}}{x_{2}}-\frac{1-e^{-\beta x_{1}}}{x_{1}}\right) .
$$

It is easily verified that the function $\left(1-e^{-\beta x}\right) / x$ is strictly decreasing in $x$ for all real $x$ and $\beta$. Hence, it follows that the right hand side of (A.21) is positive and therefore $G\left(\beta \mid x_{1}, x_{2}\right)$ is strictly decreasing in $\beta$. Thus, (A.20) has at most one possible solution for $\beta$. Then (A.20) implies that also $\gamma_{3}$ is identified. Furthermore, we realize from (A.17) that also $\gamma_{1}$ is identified. Consequently, it follows that $v(C, 0)$ is also identified. Let

$$
v^{*}(C, h)=\exp \left(\gamma_{1}\left(C^{\alpha}-1\right) / \alpha+\gamma_{3}\left(C^{\alpha}-1\right)\left((1-h / M)^{\beta}-1\right) / \alpha \beta\right) .
$$

From the results achieved above we have that $v^{*}(C, h)$ is identified. Note also that under the generalized Box-Cox functional form assumption we can express $v(C, h)$ as

$$
v(C, h)=v^{*}(C, h) \exp \left(\gamma_{2}\left((1-h / M)^{\beta}-1\right) / \beta\right)
$$

where $\gamma_{2}$ is unknown. Similarly to (A.15) it follows that $g_{2}(w \mid h)$ is identified and can be expressed as

$$
g_{2}(w \mid h)=\frac{\varphi(h, w \mid I) / v^{*}(f(h w, I), h)}{\int_{0}^{\infty}\left(\varphi(h, y \mid I) / v^{*}(f(h y, I), h)\right) d y} .
$$


It remains to show that $\gamma_{2}, \theta$ and $g_{1}(h)$ are identified. From (2.2a, b) and (A.23) we get that

$$
\begin{aligned}
& \theta g_{1}(h) \exp \left(\gamma_{2}\left((1-h / M)^{\beta}-1\right) / \beta\right)=\frac{\varphi(h, w \mid I) v(f(0, I), 0)}{\left.\varphi(0 \mid I) v^{*}(f(h w, I), h)\right)} \cdot \frac{1}{g_{2}(w \mid h)} \\
& =\frac{v^{*}(f(0, I), 0) \int_{0}^{\infty}\left(\varphi(h, y \mid I) / v^{*}(f(h y, I), h)\right) d y}{\varphi(0 \mid I)} .
\end{aligned}
$$

The right hand side of (A.24) is known. By assumption 6, we can find $h_{1}$ and $h_{2}$ in $D$ be hours of work such that $g_{1}\left(h_{1}\right)=g_{1}\left(h_{2}\right)$ which together with (A.24) imply that

$$
\log \left(\frac{\left.\varphi\left(h_{1}, w \mid I\right) v^{*}\left(f\left(h_{2} w, I\right), h_{2}\right)\right) g_{2}\left(w \mid h_{2}\right)}{\left.\varphi\left(h_{2}, w \mid I\right) v^{*}\left(f\left(h_{1} w, I\right), h_{1}\right)\right) g_{2}\left(w \mid h_{1}\right)}\right)=\gamma_{2} \frac{\left(1-h_{1} / M\right)^{\beta}-\left(1-h_{2} / M\right)^{\beta}}{\beta}
$$

which identifies $\gamma_{2}$ since $\beta$ is known. But then (A.24) implies that $\theta g_{1}(h)$ is identified and we can express $\theta g_{1}(h)$ by means of (A.24). Since $g_{1}(h)$ has the property of a probability mass function and of which the sum is equal to one we can determine $\theta$ by summing the formula for $\theta g_{1}(h)$ over all positive value of $h$. Hence, the proof is complete.

Q.E.D.

Before we embark on the proof of Theorem 5 we need the following result.

\section{Lemma 1:}

Assume that Assumption 3 holds and let the function $\kappa(C, I)$ be determined by $f(\kappa(C, I), I)=C$. Then, for $C^{\prime} \in\left(C_{0}, \infty\right)$ and $h \in D$ one can find constants $C^{*} \in\left[C_{0}, \infty\right)$ and $h^{*} \in D$ such that that there exists a positive solution $I=I^{*}(C, h)$ (say) of the equation $C^{*}=f\left(h^{*} \kappa(C, I) / h, I\right)$ for $C$ in a suitable open neighborhood of $C^{\prime}$.

\section{Proof of Lemma 1:}

Note that under Assumption 3, $\kappa(C, I)$ is well-defined and continuous. Moreover, $\kappa(C, I)$ is strictly increasing in $C$ and strictly decreasing in $I$. Evidently, solving the equation $C^{*}=f\left(h^{*} \kappa(C, I) h^{-1}, I\right)$ for $I$ is equivalent to solving

$$
\frac{\kappa(C, I)}{h}=\frac{\kappa\left(C^{*}, I\right)}{h^{*}}
$$


for $I$ because the latter equation implies that

$$
f\left(h^{*} \kappa(C, I) h^{-1}, I\right)=f\left(\kappa\left(C^{*}, I\right), I\right)=C^{*} .
$$

The last equality above follows from the definition of $\kappa(C, I)$.

Now consider the case when $h<\max \{h \mid h \in D\}$. In this case, we can choose $h^{*}=\max \{h \mid h \in D\}$. Define $I_{0}(C)$ by $\kappa\left(C, I_{0}(C)\right)=0$. For all $C^{*}>C$, we have $\kappa\left(C^{*}, I_{0}(C)\right)>\kappa\left(C, I_{0}(C)\right)=0$. Hence,

$$
\frac{\kappa\left(C^{*}, I_{0}(C)\right)}{h^{*}}-\frac{\kappa\left(C, I_{0}(C)\right)}{h}=\frac{\kappa\left(C^{*}, I_{0}(C)\right)}{h^{*}}>\frac{\kappa\left(C, I_{0}(C)\right)}{h^{*}}=0 .
$$

When $C^{*}>C$ we also have that $\kappa\left(C^{*}, 0\right)>\kappa(C, 0)$. But since $h<h^{*}$ and $\kappa(C, 0)$ is continuous in $C$, one can still choose a suitable $C^{*}>C$ such that

$$
\frac{\kappa\left(C^{*}, 0\right)}{h^{*}}<\frac{\kappa(C, 0)}{h} .
$$

Since $\kappa(C, I)$ is continuous in $I$ as well, (A.26) and (A.27) imply that (A.25) has a positive solution $I^{*}(C, h) \in\left(0, I_{0}(C)\right)$. It is easy to see that when we vary $C$ within a small neighborhood the choice of $\left(C^{*}, h^{*}\right)$ can be kept fixed.

In the case where $h=\max \{h \mid h \in D\}$, we can let $h^{*}=\min \{h \mid h \in D\}$, and similarly to the case above we can find $C^{*}<C$ such that

$$
\frac{\kappa\left(C^{*}, 0\right)}{h^{*}}>\frac{\kappa(C, 0)}{h}
$$

Since $C^{*}<C$, we have

$$
\frac{\kappa\left(C^{*}, I_{0}\left(C^{*}\right)\right)}{h^{*}}<\frac{\kappa\left(C, I_{0}\left(C^{*}\right)\right)}{h} .
$$

Since the two latter inequalities are similar to (A.26) and (A.27) it follows that (A.25) has a positive solution also in this case. This concludes the proof.

Q.E.D.

\section{Proof of Theorem 5:}

Case (i):

Consider first the case where $\xi(z)$ is distributed according to a continuous distribution with density $g_{\xi}($.$) . Under Assumption 7, we get from (2.3a) that$

(A.28) $\varphi(h, w \mid I, X)=v(f(w h, I), h) g_{1}(h) E_{\eta}\left(R(a+X b+\eta, I) g_{\xi}(\log w-X b-a-\eta)\right) / w$ 
for positive $h$, where

$$
R(u, I)=\frac{\theta(u)}{v(f(0, I), 0)+\sum_{r \in D} \int_{-\infty}^{\infty} v\left(f\left(r e^{z}, I\right), r\right) \theta(u) g_{1}(r) g_{\xi}(z-u) d z} .
$$

For any feasible combination $w>0, h \in D$ and $I \geq 0$, such that $C=f(w h, I) \geq C_{0}$, we have from Lemma 1 that one can find constants $C^{*}$ and $h^{*} \neq h$ such that the function $I^{*}(C, h)$ (depending also on $\left.C^{*}, h^{*}\right)$ defined implicitly by $f\left(h^{*} \kappa\left(C, I^{*}(C, h)\right) / h, I^{*}(C, h)\right)=C^{*}$ exists and is non-negative. From (A.28) it follows that

$$
\frac{\varphi(h, w \mid I, X)}{\varphi\left(h^{*}, w \mid I, X\right)}=\frac{v(f(w h, I), h) g_{1}(h)}{v\left(f\left(w h^{*}, I\right), h^{*}\right) g_{1}\left(h^{*}\right)} .
$$

Rewrite $w=\kappa(C, I) / h$ and it follows that (A.30) yields

$$
v(C, h) g_{1}(h)=v\left(f\left(h^{*} \kappa(C, I) / h, I\right), h^{*}\right) g_{1}\left(h^{*}\right) \frac{\varphi(h, \kappa(C, I) / h \mid I, X)}{\varphi\left(h^{*}, \kappa(C, I) / h \mid I, X\right)} .
$$

Since the left hand side of (A.31) does not depend on $I$, we can choose $I=I^{*}(C, h)$. Note that $f\left(h^{*} \kappa\left(C, I^{*}(C, h)\right) / h, I^{*}(C, h)\right)=C^{*}$ consequently, (A.31) yields

$$
v(C, h) g_{1}(h)=v\left(C^{*}, h^{*}\right) g_{1}\left(h^{*}\right) \cdot \frac{\varphi\left(h, \kappa\left(C, I^{*}(C, h)\right) / h \mid I^{*}(C, h), X\right)}{\varphi\left(h^{*}, \kappa\left(C, I^{*}(C, h)\right) / h \mid I^{*}(C, h), X\right)}
$$

for positive hours of work. Since $C^{*}$ and $h^{*}$ are constants we realize that the right hand side of (A.32) is known apart from the constant $v\left(C^{*}, h^{*}\right) g_{1}\left(h^{*}\right)$. Hence, $v(C, h) g_{1}(h)$ is identified apart from a constant but $g_{1}(h)$ cannot be identified. In other words for $h>0$ we can write $v(C, h)=\tilde{v}(C, h) \delta(h)$ where $\tilde{v}(C, h)$ is known whereas $\delta(h)$ is an unknown function of $h$.

It remains to prove identification for the parameters $a, b$, the distributions of $\xi(z), \eta$ and the function $\theta(\cdot)$. To this end we shall apply the Fourier transformation technique. Let $z=\log w$, we note that (A.28) implies that for $h>0, z \in R$

$$
\frac{e^{z} \varphi\left(h, e^{z} \mid I, X\right)}{\tilde{v}\left(f\left(e^{z} h, I\right), h\right)}=c(h) E_{\eta}\left(R(a+X b+\eta, I) g_{\xi}(z-X b-a-\eta)\right)
$$

where the left hand side of (A.33) is known, $c(h)=\delta(h) g_{1}(h)$ is an unknown positive function . Because $\tilde{v}(C, h)$ is known the left hand side of (A.33) is known. For $h \in D$ and $I>0$ let 


$$
\Lambda(\lambda, s \mid h, I)=\log \left(\int_{-\infty}^{\infty} \int_{-\infty}^{\infty} \frac{e^{z} \varphi\left(h, e^{z} \mid I, X\right)}{\tilde{v}\left(f\left(h e^{z}, I\right), h\right)} e^{i \lambda z+i s X} d z d X\right) .
$$

By Assumption 8, $\Lambda(\lambda, s \mid h, I)$ exists and is twice continuously differentiable with respect to $\lambda$ and $s$. From (A.33) it follows that $\Lambda(\lambda, s \mid h, I)$ must satisfy

$$
\begin{aligned}
\exp (\Lambda(\lambda, s \mid h, I)) & =c(h) \int_{-\infty}^{\infty} \int_{-\infty}^{\infty} E_{\eta}\left(g_{\xi}(z-X b-a-\eta) R(a+X b+\eta, I)\right) e^{i \lambda z} e^{i s X} d z d X \\
& =c(h) E_{\eta} \int_{-\infty}^{\infty} \int_{-\infty}^{\infty} g_{\xi}(z-X b-a-\eta) R(a+X b+\eta, I) e^{i \lambda z} e^{i s X} d z d X .
\end{aligned}
$$

Consider the integral

$$
\int_{-\infty}^{\infty} \int_{-\infty}^{\infty} g_{\xi}(z-X b-a-\eta) R(a+X b+\eta, I) e^{i \lambda z} e^{i s X} d z d X
$$

for given $\eta$. Next, make the change-of-variable, $(X, z) \rightarrow(u, y)$ where $u=a+X b+\eta$ and $y=z-X b-a-\eta$, which yields $X=(u-a-\eta) / b$ and $z=y+u$. The corresponding Jacobian of this transformation has absolute value equal to $|b|^{-1}$. We therefore get for given $\eta$, that

$$
\begin{aligned}
& \int_{-\infty}^{\infty} \int_{-\infty}^{\infty} g_{\xi}(z-X b-a-\eta) R(a+X b+\eta, I) e^{i \lambda z} e^{i s X} d z d X \\
= & |b|^{-1} e^{-i s(a+\eta) / b} \int_{-\infty}^{\infty} g_{\xi}(y) e^{i \lambda y} d y \cdot \int_{-\infty}^{\infty} R(u, I) e^{i(\lambda+s / b) u} d u .
\end{aligned}
$$

Define the following Fourier transforms

$$
\hat{g}_{\eta}(s)=E e^{i s \eta}, \hat{g}_{\xi}(\lambda)=\int_{-\infty}^{\infty} e^{i \lambda y} g_{\xi}(y) d y \quad \text { and } \quad \hat{R}(s \mid I)=\int_{-\infty}^{\infty} e^{i s u} R(u, I) d u .
$$

From the equations above it follows that

$$
\begin{aligned}
\exp (\Lambda(\lambda, s \mid h, I)) & =c(h) \cdot E_{\eta} \int_{-\infty}^{\infty} \int_{-\infty}^{\infty} g_{\xi}(z-X b-a-\eta) R(a+X b+\eta, I) e^{i \lambda z} e^{i s X} d z d X \\
& =c(h) \cdot|b|^{-1} e^{-i s a / b} E e^{-i s \eta / b} \int_{-\infty}^{\infty} g_{\xi}(y) e^{i \lambda y} d y \int_{-\infty}^{\infty} R(u, I) e^{i(\lambda+s / b) u} d u \\
& =c(h) \cdot|b|^{-1} e^{-i s a / b} \hat{g}_{\eta}(-s / b) \hat{g}_{\xi}(\lambda) \hat{R}(s / b+\lambda \mid I) .
\end{aligned}
$$

Since $g_{\xi}$ and $g_{\eta}$ are p.d.f. we have that $\hat{g}_{\xi}(0)=\hat{g}_{\eta}(0)=1$ and therefore we get from (A.35) that

$$
\exp (\Lambda(0, s \mid h, I))=|b|^{-1} c(h) e^{-i s a / b} \hat{g}_{\eta}(-s / b) \hat{R}(s / b \mid I)
$$


By substituting $s$ by $s+b \lambda$ in (A.36), we get that

$$
\exp (\Lambda(0, s+b \lambda \mid h, I))=|b|^{-1} c(h) e^{-i a(s / b+\lambda)} \hat{g}_{\eta}(-\lambda-s / b) \hat{R}(s / b+\lambda \mid I) .
$$

Hence, (A.35) and (A.37) yield

$$
\Lambda(\lambda, s \mid h, I)-\Lambda(0, s+b \lambda \mid h, I)=i a \lambda+\log \hat{g}_{\xi}(\lambda)+\kappa(-s / b)-\kappa(-\lambda-s / b)
$$

where $\kappa(s)=\log \hat{g}_{\eta}(s)$. By differentiating (A.38) w.r.t. $s$, we get

$$
\Lambda_{2}^{\prime}(\lambda, s \mid h, I)-\Lambda_{2}^{\prime}(0, s+b \lambda \mid h, I)=b^{-1} \kappa^{\prime}(-\lambda-s / b)-b^{-1} \kappa^{\prime}(-s / b) .
$$

By differentiating (A.39) with respect to $s$ and $\lambda$, respectively, we obtain that

$$
\Lambda_{22}^{\prime \prime}(\lambda, s \mid h, I)-\Lambda_{22}^{\prime \prime}(0, s+b \lambda \mid h, I)=-b^{-2} \kappa^{\prime \prime}(-\lambda-s / b)+b^{-2} \kappa^{\prime \prime}(-s / b)
$$

and

$$
\Lambda_{21}^{\prime \prime}(\lambda, s \mid h, I)-b \Lambda_{22}^{\prime \prime}(0, s+b \lambda \mid h, I)=-b^{-1} \kappa^{\prime \prime}(-\lambda-s / b) .
$$

By multiplying (A.40) by $b$ and subtracting (A.41) from (A.40) we get that

$$
b \Lambda_{22}^{\prime \prime}(\lambda, s \mid h, I)-\Lambda_{21}^{\prime \prime}(\lambda, s \mid h, I)=b^{-1} \kappa^{\prime \prime}(-s / b) .
$$

Since the right hand side of (A.42) is independent of $\lambda$ it follows that for any $\lambda_{1}$ and $\lambda_{2}$ that

$$
b\left(\Lambda_{22}^{\prime \prime}\left(\lambda_{2}, s \mid h, I\right)-\Lambda_{22}^{\prime \prime}\left(\lambda_{1}, s \mid h, I\right)\right)=\Lambda_{21}^{\prime \prime}\left(\lambda_{2}, s \mid h, I\right)-\Lambda_{21}^{\prime \prime}\left(\lambda_{1}, s \mid h, I\right)
$$

which identifies $b .^{13}$ Since $\xi(\mathrm{z})$ and $\eta$ both have zero mean $\hat{g}_{\xi}^{\prime}(0)=0$ and $\hat{g}_{\eta}^{\prime}(0)=0$. By differentiating (A.38) with respect to $\lambda$ and letting $\lambda=0$, it follows that

$$
\Lambda_{1}^{\prime}(0, s \mid h, I)-b \Lambda_{2}^{\prime}(0, s \mid h, I)=i a+\kappa^{\prime}(-s / b) .
$$

Then, with $s=0$, (A.43) becomes

$$
\Lambda_{1}^{\prime}(0,0 \mid h, I)-b \Lambda_{2}^{\prime}(0,0 \mid h, I)=i a .
$$

Eq. (A.44) implies that $a$ is identified. Hence, it follows from (A.43) that also $\kappa^{\prime}(\cdot)$ is identified and together with the condition $\kappa(0)=\log \hat{g}_{\eta}(0)=0, \kappa(\cdot)$ is determined. Hence, $\hat{g}_{\eta}(s)$ is identified. Moreover, (A.38) implies that $\hat{g}_{\xi}(\lambda)$ is identified. Finally, (A.36) with $s=0$ gives

$$
\exp (\Lambda(0,0 \mid h, I))=|b|^{-1} c(h) \hat{R}(0 \mid I)
$$

implying that $c(h)$ is identified up to a unknown constant $c_{0}$ (say). Subsequently, (A.36) implies that $\hat{R}(s \mid I)$ is identified up to a constant $1 / c_{0}$.

\footnotetext{
${ }^{13}$ In order for $b$ to be uniquely determined one must have that $\Lambda_{21}^{\prime \prime}(\lambda, s \mid h, I)$ (or $\Lambda_{22}^{\prime \prime}(\lambda, s \mid h, I)$ ) is not constant as a function of $\lambda$.
} 
Due to the uniqueness property of the Fourier transform we thus conclude that $g_{\eta}(\cdot)$, and $g_{\xi}(\cdot)$ are identified and $R(s, I)$ is identified up to a constant $1 / c_{0}$. Once $a, b$ and $g_{\xi}(y)$ are determined, it follows immediately from (3.3) that $g_{2}(w \mid \eta)$ is identified.

Given that $c(h)$ is identified up to a constant, $c_{0}$, it follows from the relations $c(h)=\delta(h) g_{1}(h)$ and $v(C, h) g_{1}(h)=\tilde{v}(C, h) g_{1}(h) \delta(h)=\tilde{v}(C, h) c(h)$, that $v(C, h) g_{1}(h)$ is identified up to the same constant $c_{0}$ for $h>0$. From (A.29) it follows that

$$
\frac{v(f(0, I), 0)}{\theta(u)}=\frac{1}{R(u, I)}-\sum_{r \in D} \int_{-\infty}^{\infty} \tilde{v}\left(f\left(r e^{z}, I\right), r\right) c(r) g_{\xi}(z-u) d z
$$

In view of the analysis above we know that the right hand side of (A.45) is identified up to a constant $c_{0}$. It therefore follows that $v(C, 0) / \theta(u)$ is also identified up to the constant, $c_{0}$. This is so because for any $C \geq C_{0}$ we can find a $I(C)$ (say) such that $C=f(0, I(C))$. Accordingly, it follows that $\theta(\cdot)$ and $v(C, 0)$ are identified apart from a multiplicative constant.

The special case where $\theta$ does not depend on $a+X b+\eta$ but possibly on other covariates is covered by the proof above. This completes the proof of case with continuous distributions of $\eta$ and $\xi(z)$.

Consider next the case where $\xi(z)=0$. In this case (A.34) reduces to

$$
\exp (\Lambda(\lambda, s \mid h, I))=c(h) E_{\eta} \int_{-\infty}^{\infty} R(a+X b+\eta, I) e^{i \lambda(X b+a+\eta)} e^{i s X} d X .
$$

By the change of variable; $u=a+X b+\eta$ the above equation becomes

$$
\begin{aligned}
& \exp (\Lambda(\lambda, s \mid h, I))=c(h)|b|^{-1} E_{\eta} \int_{-\infty}^{\infty} R(u, I) e^{i \lambda u} e^{i s(u-a-\eta) / b} d u \\
& =c(h)|b|^{-1} \hat{R}(\lambda+s / b, I) e^{-i s a} \hat{g}_{\eta}(-s / b) .
\end{aligned}
$$

From (A.46) it follows that

$$
\Lambda(\lambda, s \mid h, I)-\Lambda(0, s+\lambda b \mid h, I)=i a \lambda+\kappa(-s / b)-\kappa(-\lambda-s / b) .
$$

We note that (A.47) is a special case of (A.38) obtained when $\hat{g}_{\xi}(\lambda)=1$. Accordingly, it is clear that the model also is identified in this case.

The proof for the case where either $\eta$ or $\xi(z)$ or both have discrete distributions is similar. This completes the proof for case $(i)$. 
Case (ii):

Above we have proved that $v(C, h)=\tilde{v}(C, h) \delta(h)$ where $\tilde{v}(C, h)$ is known and $\delta(h)$ is an unknown function of $h$. Thus $\partial^{2} \log v(C, h) / \partial C^{2}$ is identified. Then under the generalized Box-Cox assumption (3.2), one can use the same procedure as in the proof of Theorem 4 regarding identification of $\alpha, \beta, \gamma_{1}$ and $\gamma_{3}$. (See the discussion from equation (A.18) to (A.23)). This also proves the identification of $v(C, 0)$. It therefore only remains to identify $\gamma_{2}$. Note that above we also proved that $v(C, h) g_{1}(h)$ is identified up to a constant $c_{0}$ for $h>0$. Now let $h_{1}$ and $h_{2}$ be two distinct points in $D$ such that $g_{1}\left(h_{1}\right)=g_{1}\left(h_{2}\right)$. Due to the generalized Box-Cox assumption it consequently follows that

$$
\log \left(\frac{v\left(C, h_{2}\right) g_{1}\left(h_{2}\right)}{v\left(C, h_{1}\right) g_{1}\left(h_{1}\right)}\right)=\left(\gamma_{3} \frac{C^{\alpha}-1}{\alpha}+\gamma_{2}\right)\left(\frac{\left(1-h_{2} / M\right)^{\beta}-\left(1-h_{1} / M\right)^{\beta}}{\beta}\right) .
$$

Since the left hand side of (A.48) is known and we have demonstrated that all the parameters except $\gamma_{2}$ are identified it follows that also $\gamma_{2}$ is identified. Thus, we have identified $v(C, h)$.

By using again the fact that $v(C, h) g_{1}(h)$ is identified apart from a multiplicative constant, it follows that $g_{1}(h)$ is identified apart from a constant. But this constant can be determined from the property that $g_{1}(h)$ is a probability mass function on $D$. Thus $g_{1}(h)$ is identified and $v(C, h) g_{1}(h)$ is identified. This implies that $c_{0}$ is identified. Thus, (A.45) implies that $\theta(\cdot)$ is identified. The proof is now complete.

Q.E.D. 


\section{Appendix B.}

\section{Data Description}

The data are obtained by merging the Labor force survey 1997 with two different register data sets that contain additional information about incomes, family composition, children and education. The concepts of the Labor force survey are in accordance with official statistics from Statistics Norway and recommendations given by ILO. Here we note that persons are asked about their attachment to the labor market during a particular week.

Information about actual and formal working time in main as well as second job and background variables such as demographic characteristics and occupation is included in the Labor force survey. Conditional on labor market participation, respondents are asked whether they consider themselves as self-employed or employee. Households where one of the adults has income from self-employment higher than NOK 80000 are excluded. A person is defined as working if he works at least one hour per week. Hours of work is measured as the sum of formal hours of work in the main and the second job (if the individual have a second job). If this information is missing and the respondent is participating in the labor market, information about actual working time is used.

Information on education is obtained from the National Education database, a register database that can be linked to the Labor force survey using the system with personal identification numbers.

Whereas the Labor force survey yields detailed information about employment and hours of work, it does not provide information about annual labor incomes that can be used in the calculations of (average) gross wage rates, and non-labor income. To obtain this information we apply the Tax Return Register (includes more detailed information about employee income, self-employment income, taxable pensions etc.) These data can be linked to the Labor force survey using personal identification numbers. Nominal hourly wage rates are measured as labor income, measures as the sum of labor income from the main and the second job (if the individual has a second job) divided by (formal) total annual hours of work (for main -and second job). Households where one of the adults has hours of work higher than 80, or wage rate less than NOK 50 or higher than NOK 400 are excluded. Experience (potential experience) is defined as age minus years of schooling minus 7. 
The sample we use only includes persons with age between 26-62 years. The motivation for this is that for women below 26 years of age education is an important activity

Table B.1 Summary Statistics

\begin{tabular}{|c|c|c|c|c|c|c|}
\hline & \multicolumn{2}{|c|}{ both working } & \multicolumn{2}{|c|}{$\begin{array}{l}\text { only husband } \\
\text { working }\end{array}$} & \multicolumn{2}{|c|}{ only wife working } \\
\hline & mean & std & mean & std & mean & std \\
\hline \multicolumn{7}{|l|}{ Men } \\
\hline Age & 45.06 & 8.44 & 44.06 & 9.41 & 50.40 & 9.91 \\
\hline Education & 12.58 & 2.89 & 12.26 & 2.71 & 11.80 & 3.03 \\
\hline Experience & 25.48 & 9.14 & 24.80 & 9.99 & 31.60 & 11.08 \\
\hline non-labor income & 6320 & 12032 & 10796 & 15543 & 30604 & 30395 \\
\hline wage rate & 153.82 & 52.98 & 169.11 & 65.47 & & \\
\hline weekly hours of work & 38.43 & 5.38 & 39.16 & 5.91 & & \\
\hline \multicolumn{7}{|l|}{ Women } \\
\hline Age & 42.75 & 8.39 & 41.68 & 9.70 & 47.40 & 7.86 \\
\hline Education & 12.10 & 2.72 & 10.87 & 2.26 & 11.80 & 2.86 \\
\hline Experience & 23.65 & 9.33 & 23.81 & 10.71 & 28.60 & 10.01 \\
\hline non-labor income & 17655 & 16558 & 27689 & 21701 & 13848 & 14219 \\
\hline wage rate & 120.12 & 37.79 & & & 22.82 & 17.98 \\
\hline weekly hours of work & 30.45 & 8.93 & & & 29.78 & 12.06 \\
\hline No of Children 0-6 & 0.37 & 0.68 & 0.70 & 0.89 & 0.20 & 0.45 \\
\hline No of children 7-18 & 0.85 & 0.97 & 0.94 & 0.97 & 1.00 & 0.71 \\
\hline Number of Households & 2254 & & 256 & & 5 & \\
\hline
\end{tabular}

and for those more than 62 years of age early retirement is rather frequent. The number of children includes all children with age less than 19. In Table B.1 we report the summary statistics for the sample used in estimating the labor supply model. 


\section{Appendix C.}

\section{Model for Married Couples}

The modeling framework for two-person households is completely similar to the case for single individual households. Let $U\left(C, h_{F}, h_{M}, z\right)$ denote the utility function of a household, where $h_{F}$ and $h_{M}$ are hours of work for female and male and $z=\left(z_{F}, z_{M}\right)$ indexes the combination of jobs for the female and male in the household, respectively. Similarly to Assumption 1, assume that $U\left(C, h_{F}, h_{M}, z\right)=v\left(C, h_{F}, h_{M}\right) \varepsilon(z)$. The budget constraint in this case can be written as

$$
C=f\left(h_{F} w_{F}, h_{M} w_{M}, I\right)
$$

where $w_{F}$ and $w_{M}$ are the respective wage rates for female and male and $f(\cdot)$ is the function that transforms gross income to disposable income for the household. Analogous to Assumption 2 we assume that for positive $z_{F}$ and $z_{M},\left\{\varepsilon\left(z_{F}, z_{M}\right), z_{F}, z_{M}=1,2, \ldots\right\}$ is an enumeration of the points of a Poisson process on $(0, \infty)$ with intensity measure $\theta_{F} \theta_{M} \varepsilon^{-2} d \varepsilon$. Similarly, when $z_{F}>0, z_{M}<0,\left\{\varepsilon\left(z_{F}, z_{M}\right), z_{F}=1,2, \ldots, z_{M}=\ldots-2,-1\right\}$ is an enumeration of the realizations of a Poisson process with intensity measure $\theta_{F} \varepsilon^{-2} d \varepsilon$. The latter Poisson process corresponds to the distribution of taste shifters across combinations of available jobs for the female and non-market opportunities for the male. The taste-shifters for the case where $z_{F}<0, z_{M}>0$ are also realizations of a Poisson process with intensity measure $\theta_{M} \varepsilon^{-2} d \varepsilon$. Finally, the case where $z_{F}<0, z_{M}<0$ are realizations of a Poisson process with intensity measure $\varepsilon^{-2} d \varepsilon$. All four Poisson processes are assumed to be independent. The combinations of offered hours and wage rates to the female are assumed independent of the offered hours and wage rates to the male. The respective opportunity densities given by $g_{1 F}\left(h_{F}\right) g_{2 F}\left(w_{F} \mid h_{F}\right)$ and $g_{1 M}\left(h_{F}\right) g_{2 M}\left(w_{M} \mid h_{M}\right)$.

Let $\varphi\left(h_{F}, w_{F}, h_{M}, w_{M} \mid I\right)$ be the joint density of hours of work and wage rate for female and male in the household, given household non-labor income $I$. Then, model (2.2a, b) can be generalized to 
$\varphi\left(h_{F}, w_{F}, h_{M}, w_{M} \mid I\right)=\frac{v\left(f\left(w_{F} h_{F}, w_{M} h_{M}, I\right), h_{F}, h_{M}\right) \theta_{F} g_{1 F}\left(h_{F}\right) g_{2 F}\left(w_{F} \mid h_{F}\right) \theta_{M} g_{1 M}\left(h_{F}\right) g_{2 M}\left(w_{M} \mid h_{M}\right)}{M}$.

for $h_{F}>0, h_{M}>0$, and

$$
\varphi(0,0,0,0 \mid I)=\frac{v(f(0,0, I), 0,0)}{M}
$$

for $h_{F}=0$ and $h_{M}=0$, where

$$
\begin{aligned}
& M(I)=v(f(0,0, I), 0,0)+\theta_{M} \sum_{x \in D} \int_{0}^{\infty} v(f(0, x y, I), 0, x) g_{1 M}(x) g_{2 M}(y \mid x) d y \\
& +\theta_{F} \sum_{x \in D} \int_{0}^{\infty} v(f(x y, 0, I), x, 0) g_{1 F}(x) g_{2 F}(y \mid x) d y \\
& +\theta_{F} \theta_{M} \sum_{x_{1}, x_{2} \in D} \int_{0}^{\infty} \int_{0}^{\infty} v\left(f\left(x_{1} y_{1}, x_{2} y_{2}, I\right), x_{1}, x_{2}\right) g_{1 F}\left(x_{1}\right) g_{2 F}\left(y_{1} \mid x_{1}\right) g_{1 M}\left(x_{2}\right) g_{2 M}\left(y_{2} \mid x_{2}\right) d y_{1} d y_{2}
\end{aligned}
$$

Generalization of model (2.4a,b) to the case of married couples is similar.

\section{Empirical model specification:}

The job availability indexes $\theta_{k}, k=M, F$, are assumed to depend on the wages solely through the amount of schooling. Specifically, we assume that

$$
\log \theta_{k}=f_{k 1}+f_{k 2} S,
$$

where $S$ is the length of education. Furthermore, we specify the deterministic part of the utility function $v(\cdot)$ to be of the form

$$
\begin{array}{r}
\log v\left(C, h_{F}, h_{M}\right)=\gamma_{1} \frac{\left(10^{-4}\left(C-C_{0}\right)^{\alpha_{1}}-1\right)}{\alpha_{1}}+\gamma_{2 M} \frac{\left(\left(1-h_{M} / M\right)^{\beta_{M}}-1\right)}{\beta_{M}}+\gamma_{2 F} \frac{\left(\left(1-h_{F} / M\right)^{\beta_{F}}-1\right)}{\beta_{F}} \\
+\mu \frac{\left(\left(1-h_{M} / M\right)^{\beta_{M}}-1\right)}{\beta_{M}} \frac{\left(\left(1-h_{F} / M\right)^{\beta_{F}}-1\right)}{\beta_{F}}
\end{array}
$$

where

$$
\begin{array}{r}
\gamma_{2 F}=\alpha_{5}+\alpha_{6} \log A_{F}+\alpha_{7}\left(\log A_{F}\right)^{2}+\alpha_{8} C U 6+\alpha_{9} C O 6, \\
\gamma_{2 M}=\alpha_{10}+\alpha_{11} \log A_{M}+\alpha_{12}\left(\log A_{M}\right)^{2}+\alpha_{13} C U 6+\alpha_{14} C O 6,
\end{array}
$$


$C_{0}$ is the subsistence level of disposable income, $A_{k}$ is the age for gender $k, k=F, M$,

divided by $10^{14}$, CU6 and CO6 are the number of children below or equal to and above the age of six respectively, $C$ is given by the budget constraint similarly to the case of single household. $M$ is the maximum available hours of work, which is set as 3650 hours for a year. This corresponds to about 14 hours per day reserved for sleep and rest. We have chosen $C_{0}$ to be approximately NOK 40,000 $\sqrt{N}$, where $N$ is the number of persons in the household.

Disposable income, $C$, is measured as the sum of the annual household wage incomes after tax, household capital income after tax, and child allowances. Furthermore, it is assumed that $g_{2 F}\left(w_{F} \mid h_{F}\right)=g_{2 F}\left(w_{F}\right)$ and $g_{2 M}\left(w_{M} \mid h_{M}\right)=g_{2 M}\left(w_{M}\right)$.

Table C.1. Estimates of wage equations, men and women, 1997

\begin{tabular}{lrrrrrrrrr}
\hline & \multicolumn{4}{c}{ Men } & \multicolumn{4}{c}{ Women } \\
& \multicolumn{1}{c}{ Standard Mincer } & \multicolumn{2}{c}{ Selection corrected } & \multicolumn{2}{c}{ Standard Mincer } & \multicolumn{2}{c}{ Selection corrected } \\
& Estimate & Std & Estimate & Std & Estimate & Std & Estimate & Std \\
Constant & 4.112 & 0.030 & 4.117 & 0.035 & 4.132 & 0.030 & 4.149 & 0.036 \\
Education in years & 0.043 & 0.002 & 0.043 & 0.002 & 0.038 & 0.002 & 0.037 & 0.002 \\
Experience in years/10 & 0.217 & 0.018 & 0.217 & 0.018 & 0.135 & 0.016 & 0.129 & 0.018 \\
(Experience in years/10) & -0.036 & 0.004 & -0.036 & 0.004 & -0.021 & 0.003 & -0.020 & 0.004 \\
Dummy for married & 0.053 & 0.009 & 0.052 & 0.010 & -0.022 & 0.008 & -0.019 & 0.009 \\
logP & & & 0.104 & 0.442 & & & 0.038 & 0.044 \\
Std of Error term & 0.302 & & 0.302 & & 0.275 & & 0.275 & 5072 \\
No. Observations & 5446 & & 5446 & & 5072 & & 0.10 & & 0.10 \\
R square & 0.15 & & 0.15 & & & 0.10 & \\
\hline
\end{tabular}

${ }^{14}$ To avoid possible multi-colinearity, we have subtracted the mean of $\log \left(A_{k}\right)$ in estimation. 
Table C.2. Estimates of the parameters of the utility function. Married couples, 1997.

\begin{tabular}{|c|c|c|c|c|}
\hline Preferences: & \multicolumn{2}{|c|}{$\begin{array}{c}\text { Model } 1 \\
\text { (wage varies only over jobs) }\end{array}$} & \multicolumn{2}{|c|}{$\begin{array}{c}\text { Model 2 } \\
\text { (wage varies only across individuals) }\end{array}$} \\
\hline \multicolumn{5}{|l|}{ Consumption } \\
\hline Exponent & 0.460 & 0.087 & 0.595 & 0.058 \\
\hline Scale $10^{\sqcup 4}$ & 1.488 & 0.444 & 2.299 & 0.485 \\
\hline Subsistence* & $40000 \sqrt{N}$ & & $40000 \sqrt{N}$ & \\
\hline \multicolumn{5}{|l|}{ Female leisure } \\
\hline Exponent & -2.622 & 0.239 & -0.710 & 0.204 \\
\hline Constant & 1.365 & 0.839 & 7.365 & 1.978 \\
\hline $\log (\text { age } / 10)^{* *}$ & 1.886 & 1.060 & 2.730 & 0.638 \\
\hline Log(age/10) squared & 7.859 & 3.901 & 7.008 & 1.980 \\
\hline No. children below or equal 6 years & 0.165 & 0.176 & 0.915 & 0.187 \\
\hline No. children above 6 years & 0.029 & 0.106 & 0.726 & 0.139 \\
\hline \multicolumn{5}{|l|}{ Male leisure } \\
\hline Exponent & -0.454 & 0.562 & 0.495 & 0.212 \\
\hline Constant & 1.365 & 0.839 & 10.876 & 3.135 \\
\hline Log(age/10)** & 1.886 & 1.060 & 4.362 & 1.609 \\
\hline Log(age/10) squared & 7.859 & 3.901 & 16.030 & 5.518 \\
\hline No. children below or equal 6 years & 0.165 & 0.176 & 0.379 & 0.374 \\
\hline No. children above 6 years & 0.029 & 0.106 & 0.037 & 0.262 \\
\hline Leisure interaction & 0.228 & 0.163 & 3.247 & 1.342 \\
\hline \multicolumn{5}{|l|}{ The parameters $\log \theta_{F}$; } \\
\hline Constant & -3.420 & 0.346 & -3.264 & 0.417 \\
\hline Education/10 & 1.621 & 0.303 & 1.195 & 0.362 \\
\hline \multicolumn{5}{|l|}{ The parameters $\log \theta_{M}$} \\
\hline Constant & -1.181 & 2.184 & -0.247 & 2.398 \\
\hline Education/10 & -0.309 & 1.735 & -0.843 & 1.886 \\
\hline \multicolumn{5}{|l|}{$\begin{array}{l}\text { Opportunity density of offered } \\
\text { hours }\end{array}$} \\
\hline Male full-time peak & 3.121 & 0.101 & 3.072 & 0.109 \\
\hline Female full-time peak & 1.249 & 0.082 & 1.340 & 0.078 \\
\hline Male part-time peak & 0.231 & 0.276 & 0.384 & 0.282 \\
\hline Female part-time peak & 0.467 & 0.067 & 0.412 & 0.067 \\
\hline Number of Observations & 2515 & & 2515 & \\
\hline Log likelihood & -5309.44 & & -5242.71 & \\
\hline
\end{tabular}

${ }^{*} \mathrm{~N}$ is the size of the household. ${ }^{* *}$ To avoid potential multi-colinearity, we have used the deviation from the mean of $\log ($ age/10). 


\section{Appendix D}

\section{Simulation of changes in the opportunity distribution of offered hours of work}

Recall that in our framework, $g_{1 F}(h)$ represents the proportion of jobs with hours of work, $h$, that are available to the wife, whereas the parameter $\theta_{F}$ is a measure of job availability to the wife. Note that there are two peaks in the estimated offered hour distribution when $h=h_{p}$ (part-time) and $h=h_{f}$ (full-time). The proposed reform can be interpreted as a change in the opportunity distribution of hours for the women, obtained by removing the part-time peak and increasing the full-time peak (since the part-time jobs are replaced by full-time ones) while keeping the job availability unchanged (i.e. $\theta_{F}$ is unchanged). Denote the new opportunity distribution by $g_{1 F}^{*}(h)$. After the part-time peak has been removed, the offered hours are uniformly distributed apart from a peak at the full-time interval. Since there are five intervals for which the new opportunity density is constant we must have that

$$
g_{1 F}^{*}(h)=\left(1-g_{1 F}\left(h_{p}\right)-g_{1 F}\left(h_{f}\right)\right) / 5
$$

for $h \neq h_{f}$. In addition, the sum of jobs in part-time and full-time intervals is the same before and after reform:

$$
\theta_{F} g_{1 F}\left(h_{p}\right)+\theta_{F} g_{1 F}\left(h_{F}\right)=\theta_{F} g_{1 F}^{*}\left(h_{p}\right)+\theta_{F} g_{1 F}^{*}\left(h_{F}\right) .
$$

From these two equations it follows that

$$
g_{1 F}^{*}\left(h_{f}\right)=\left(6 g_{1 F}\left(h_{p}\right)+6 g_{1 F}\left(h_{f}\right)-1\right) / 5 \text {. }
$$

One can apply the model to simulate the corresponding realized labor supply distribution by replacing $g_{1 F}(h)$ with $g_{1 F}^{*}(h)$. 